\title{
Uniform flow of viscoelastic fluids past a confined falling cylinder
}

\author{
Alexandre Afonso • Manuel A. Alves • \\ Fernando T. Pinho $\cdot$ Paulo J. Oliveira
}

Received: 25 June 2007 / Accepted: 23 October 2007 / Published online: 6 February 2008

(C) Springer-Verlag 2008

\begin{abstract}
Uniform steady flow of viscoelastic fluids past a cylinder placed between two moving parallel plates is investigated numerically with a finite-volume method. This configuration is equivalent to the steady settling of a cylinder in a viscoelastic fluid, and here, a 50\% blockage ratio is considered. Five constitutive models are employed (UCM, Oldroyd-B, FENE-CR, PTT and Giesekus) to assess the effect of rheological properties on the flow kinematics and wake patterns. Simulations were carried out under creeping flow conditions, using very fine meshes, especially in the wake of the cylinder where large normal stresses
\end{abstract}

A. Afonso - M. A. Alves

Departamento de Engenharia Química, CEFT,

Faculdade de Engenharia da Universidade do Porto,

Rua Dr. Roberto Frias,

4200-465 Porto, Portugal

A. Afonso

e-mail: aafonso@fe.up.pt

M. A. Alves

e-mail: mmalves@fe.up.pt

F. T. Pinho

Universidade do Minho,

Largo do Paço,

4704-553, Braga, Portugal

F. T. Pinho $(\square)$

Centro de Estudos de Fenómenos de Transporte,

Faculdade de Engenharia da Universidade do Porto,

Rua Dr. Roberto Frias,

4200-465 Porto, Portugal

e-mail: fpinho@fe.up.pt

\section{P. J. Oliveira}

Departamento de Engenharia Electromecânica,

Unidade de Materiais Têxteis e Papeleiros,

Universidade da Beira Interior,

6201-001 Covilhã, Portugal

e-mail: pjpo@ubi.pt are observed at high Deborah numbers. Some of the results are compared with numerical data from the literature, mainly in terms of a drag coefficient, and significant discrepancies are found, especially for the constant-viscosity constitutive models. Accurate solutions could be obtained up to maximum Deborah numbers clearly in excess of those reported in the literature, especially with the PTT and FENE$\mathrm{CR}$ models. The existence or not of a negative wake is identified for each set of model parameters.

Keywords Falling cylinder - Viscoelastic $\cdot$ UCM .

Oldroyd-B · FENE-CR · PTT · Giesekus ·

Negative wake $\cdot$ Numerical simulations

\section{Introduction}

The flow of a viscoelastic fluid past a cylinder has been extensively studied both experimentally (Manero and Mena (1981), Bush (1993), McKinley et al. (1993) and Broadbent and Mena (1974)) and numerically (Bush (1993), Hu and Joseph (1990), Huang and Feng (1995), Liu et al. (1998), Oliveira et al. (1998), Fan et al. (1999), Sun et al. (1999), Alves et al. (2001), Caola et al. (2001), Owens et al. (2002), Phan-Thien and Dou (1999), Dou and Phan-Thien (2003), Kim et al. (2004, 2005a), Oliveira and Miranda (2005) and Gerritsma (2006)). The large number of works in this geometry may be explained from two major motivations: (1) it is representative of the fundamental flow dynamics of viscoelastic fluids around solid bodies, and (2) it is intrinsically related to many processes in chemical engineering, namely, flows through porous media (McKinley et al. 1993), enhanced oil recovery, composite and textile coating operations (Liu et al. 1998) and food processes. From a numerical point of view, the flow past a cylinder is 
a smooth flow because it does not introduce any geometrical singularity, in contrast, for instance, with the salient corner in entry flows. Nonetheless, the development of thin stress boundary layers on the cylinder sidewalls and especially the high normal stress developed along the rear wake centerline, remain a challenge and impose a limiting value on the Deborah number $(D e)$, at least as far as a symmetric steady flow is concerned.

Two configurations are possible for flow around a cylinder: either the cylinder is fixed relative to the confining channel walls and a planar Poiseuille flow emerges from an imposed pressure gradient, or it moves as in the case of a cylinder falling freely along the middle of a channel, which is equivalent to a uniform flow approaching a fixed cylinder with channel walls moving with the fluid. The present paper deals with the second situation which gives rise to the interesting phenomenon of "negative wakes" (Sigli and Coutanceau 1977 and Hassager 1979), to be discussed further down in this section but essentially consisting of fluid in the wake of the cylinder moving faster and in the opposite direction to the wake-generating object. In a frame of reference fixed to the cylinder, this correspond to velocities in the wake that are faster and in the same direction as the uniform approach flow, a situation that arises only with viscoelastic fluids. The nature of negative wakes is not yet well understood, and one of the motivations for this work was numerical quantification of conditions for its formation.

The case of confined flow around a fixed cylinder has been investigated more often than the settling of a cylinder in bounded or unbounded domains, and Owens et al. (2002) documented and summarized the main results in their book, focusing primarily on the Oldroyd-B model. Phan-Thien and Dou (1999) carried out simulations of confined cylinder flows with the upper-convected Maxwell (UCM), Oldroyd-B and PTT models where the flow was fully developed well upstream of the cylinder. They found negative wakes behind the cylinder for this flow at high Deborah numbers and only for the PTT model. Alves et al. (2001) implemented classical high-resolution interpolation schemes for convection (the MINMOD and SMART schemes) in the general collocated finite-volume method (FVM) procedure for viscoelastic flows developed by Oliveira et al. (1998). This implementation enhanced numerical accuracy and was tested with the benchmark problem of the flow past a confined cylinder with blockage ratio of 0.5 , using the UCM and the Oldroyd-B models. Highly refined non-orthogonal meshes were used which allowed a good comparison of the predicted drag force on the cylinder (the benchmark result) with values from other simulations in the literature (Liu et al. 1998, Fan et al. 1999, Sun et al. 1999, Caola et al. 2001, Owens et al. 2002, PhanThien and Dou 1999 and Kim et al. 2004). In particular, predictions of the drag coefficient up to $D e \approx 0.7$ were consistent with the finite element method (FEM) simulations of Fan et al. (1999). More recently, Gerritsma (2006) presented results obtained with a spectral element method and again in excellent agreement with the numerical results obtained by Fan et al. (1999) and Alves et al. (2001).

The settling of a cylinder in a confined viscoelastic fluid is still a "work in progress" in rheological engineering, and several contributions have come forward in the recent past. Huang and Feng (1995) investigated the steady settling of a cylinder through quiescent Newtonian and Oldroyd-B fluids in a vertical channel, employing FEM with the elastic-viscous stress split scheme (EVSS). For their higher blockage case (50\%), Huang and Feng (1995) predicted a negative wake at high Deborah numbers, but this unusual phenomenon was not replicated by Oliveira et al. (1998) in their numerical work based on the FVM. The predictions of Oliveira et al. (1998) for the unbounded flow case and the confined case with blockage area of 33\% were in agreement with those of Huang and Feng (1995), but discrepancies were found in the wake velocities for the higher blockage case $(50 \%)$. These discrepancies were attributed to the response of viscoelastic fluids to intense local shear and elongational flows at the proximity of the channel wall. Dou and Phan-Thien (2004) carried out simulations of the uniform flow of a viscoelastic fluid past a cylinder in a moving channel using the UCM, PTT, Oldroyd-B, and the FENE-CR models. They used a control volume finite element method (CVFEM) with a DEVSS- $\omega$ formulation under a distributed computing environment through a Parallel Virtual Machine (PVM) library. Again, a negative wake was not observed with the UCM and Oldroyd-B fluids for various retardation ratios $(\beta=0.125,0.4,0.6,0.8)$, thus corroborating the predictions of Oliveira et al. (1998) in contrast to the results of Huang and Feng (1995). However, for the PTT and FENE-CR models, a negative wake appeared at a critical $D e$. Regarding drag coefficient $\left(C_{\mathrm{D}}\right)$ predictions, the early calculations of Dou and PhanThien (2003) found a monotonic decrease of $C_{\mathrm{D}}$ with $D e$ for the FENE-CR fluid with lower extensibility parameter $\left(L^{2}=\right.$ $10)$. For a higher extensibility parameter $\left(L^{2}=100\right)$, however, they predicted a non-monotonic behaviour, with an initial decrease followed by an increase for approximately $D e>0.6$, related to the strong increase of the extensional effects. The numerical investigation of Kim et al. (2005a) focused on the comparison of negative wake generation in both uniform and Poiseuille flows past a cylinder, and on the influence of the FENE-CR model parameters upon this flow feature: viscosity ratio $(\beta)$ and polymer extensibility $\left(L^{2}\right)$. By employing a discrete elastic viscous split stress (DEVSS-G)streamline upwinding/Petrov-Galerkin (G/SUPG) formulation with an efficient iterative solution method developed for the mixed FEM by Kim et al. (2004), they found that the drag coefficient monotonically decreased for both $L^{2}=10$ and $L^{2}=$ 
100 , in contrast with the results obtained by Dou and PhanThien (2003). They also found that for all flow conditions and model parameters, the negative wake generation is more pronounced when the approach flow is uniform than when it is a Poiseuille flow.

So, for some viscoelastic fluids, the steady flow behind a cylinder is characterized by the appearance of a "negative wake" which strongly depends on fluid rheology. Negative wakes are overshoots of the streamwise velocity seen by the moving body along the centreline and have been found in the wake of cylinders, spheres and rising bubbles (Sigli and Coutanceau (1977) and Hassager (1979)). One of the most extensive early investigations of this phenomenon was the visualization of the sedimentation of a sphere in shearthinning polyacrylamide (PAA) solutions by Arigo and McKinley (1998), who also made an extensive review of the literature. Early, however, McKinley et al. (1993) had been unable to detect the appearance of negative wakes downstream of a cylinder for Boger fluids, and up to the present time, there is no experimental evidence of negative wake formation with constant viscosity elastic fluids. Negative wakes in the settling sphere problem have also been extensively studied using numerical methods (Jin et al. 1991, Zheng et al. 1991 and Bush 1994). These works lead to the general conclusion that both shear thinning and elastic effects are necessary for the formation of a negative wake, but the numerical simulations by Satrape and Crochet (1994) and Harlen (2002) showed that a negative wake can also be present for constant viscosity elastic fluids, such as those represented by the FENE-CR model. The predicted velocity profiles along the centreline downstream of the sphere wake were intimately linked to the extensional properties of the constitutive model, especially the extensibility parameter $L^{2}$. For small values of $L^{2}$, a negative wake was predicted even at low De values. On the other hand, increasing the strainhardening character of the model (by increasing $L^{2}$ ), the negative wake was reduced and eventually eliminated.

The physical conditions under which the negative wake arises and the mechanisms involved in its formation are still not fully understood in spite of several studies that have focused on these issues. In the sphere/cylinder geometries, Bush (1994) attributed the negative wake behaviour to the relative proportion between elongational stresses in the downstream region and fluid elasticity, and suggested that the upstream shift in streamlines and the formation of negative wake are a result of having a Deborah number much greater than the Trouton ratio. For the sphere-flow case, Harlen (2002) proposed that the origin of negative wake was related to the circumferential gradient of shear stress along the centreline and in particular with the competition of shear and normal stress distributions. Based on the suggestions of Bush (1994) and invoking also arguments later used by Harlen (2002), Arigo and McKinley
(1998) proposed a criterion for the formation of negative wake based on the ratio of axial tensile to shear stresses. Dou and Phan-Thien (2004) also studied this phenomenon numerically and proposed a different criterion, this time based on the ratio between the gradient and the magnitude of elongational viscosity, to predict the critical $D e$ marking the onset of negative wake for several constitutive models (PTT, FENE-CR, FENE-P and Giesekus models).

Identifying the conditions that lead to the onset of a negative wake is one of the motivations for the present work. In terms of outcome, this study leads to two important contributions: (1) clarify the significant discrepancies in the predicted drag coefficient of a FENE-CR fluid obtained by Dou and Phan-Thien (2003) and Kim et al. (2005a), and (2) confirmation of some sets of results available in the literature which were obtained by different numerical methods. It should thus be clear that the present work is essentially numerical and aims at clarifying the conditions under which negative wakes are formed for the most common differential constitutive models, and giving reliable data for the drag coefficient on the falling cylinder. A detailed matching of existing experimental measurements requires close fitting of the rheology of the fluids, most certainly through the incorporation of a multimode model, and as such it should be left for a future work.

The paper is organized as follows: in the "Governing equations and numerical method" and in the "Problem description and computational meshes" sections, we briefly describe the general flow problem, present the governing equations and outline the numerical method used to simulate the settling of the cylinder in a confined viscoelastic fluid. In the "Results and discussion" section, results are presented for all constitutive models, encompassing the predicted drag coefficient, detailed profiles of velocity and stress components in the vicinity of the cylinder and stability criteria. A summary of the main findings closes the paper in the "Conclusions" section.

\section{Governing equations and numerical method}

The flow is assumed to be steady, laminar, and the fluid is incompressible. The governing equations are those expressing conservation of mass:

$$
\nabla \bullet \mathbf{u}=0
$$

and momentum balance:

$\rho \frac{\partial \mathbf{u}}{\partial t}+\rho \nabla \bullet \mathbf{u u}=-\nabla p+\nabla \bullet \boldsymbol{\tau}+\eta_{\mathrm{s}} \nabla^{2} \mathbf{u}$

where $\mathbf{u}$ is the velocity vector, $p$ the pressure, $t$ the time, $\rho$ the fluid density, $\eta_{\mathrm{s}}$ the Newtonian solvent viscosity and $\tau$ the polymeric extra stress contribution. Five constitutive 
equations are employed: the upper-convected Maxwell model (UCM), the Oldroyd-B model (Bird et al. 1987), the linear form of the simplified PTT model (Phan-Thien and Tanner 1977 and Phan-Thien 1978), the modified FENE-CR model (Chilcott and Rallison 1988) and the Giesekus model (Giesekus 1982). For an isothermal flow, these five rheological equations of state can be written in a compact form as:

$$
\begin{aligned}
& P(\operatorname{tr} \boldsymbol{\tau}) \boldsymbol{\tau}+\frac{\lambda}{F}\left[\frac{\partial \boldsymbol{\tau}}{\partial t}+\mathbf{u} \bullet \nabla \boldsymbol{\tau}-\nabla \mathbf{u} \bullet \boldsymbol{\tau}-\nabla \mathbf{u}^{T} \bullet \boldsymbol{\tau}\right] \\
& +\mathbf{G}=2 \eta_{\mathrm{p}} \mathbf{D} \\
& F=\frac{L^{2}+\left(\lambda / \eta_{P}\right) \operatorname{tr}(\boldsymbol{\tau})}{L^{2}-3} \\
& P(\operatorname{tr} \boldsymbol{\tau})=1+\frac{\varepsilon \lambda}{\eta_{\mathrm{P}}} \operatorname{tr}(\boldsymbol{\tau})
\end{aligned}
$$

$$
\mathbf{G}=\alpha \boldsymbol{\tau}^{2}
$$

where $\lambda$ is a relaxation time, $\eta_{\mathrm{p}}$ is the polymer viscosity coefficient, $\mathbf{D}$ is the rate of deformation tensor and $F$ is the stretch function that depends on the extensibility parameter $L^{2}$, representing the ratio of the maximum to equilibrium average dumbbell extensions. The stress coefficient function, $P(\operatorname{tr} \boldsymbol{\tau})$, depends on the trace of $\boldsymbol{\tau}$, and $\mathbf{G}$ is the nonlinear term of the Giesekus model, with $\alpha$ representing a dimensionless "mobility factor". The stress coefficient function of the PTT model, $P(\operatorname{tr} \tau)$, introduces the dimensionless parameter $\varepsilon$ which is closely related to the steady-state elongational viscosity in extensional flows $\left(\eta_{\mathrm{E}} \backsim 1 / \varepsilon\right.$ for low $\left.\varepsilon\right)$.

The viscosity ratio is defined here as the ratio of the solvent to total viscosities (note that in some works, $\beta$ is instead defined as $\eta_{\mathrm{p}} / \eta_{0}$ (e.g. Dou and Phan-Thien 2003 and Kim et al. 2005a):

$\beta=\frac{\eta_{\mathrm{s}}}{\eta_{\mathrm{s}}+\eta_{\mathrm{P}}}=\frac{\eta_{\mathrm{s}}}{\eta_{0}}$

A specific constitutive model can be chosen by an appropriate selection of parameters $\alpha, L^{2}, \varepsilon$ and $\beta$, and the range of all parameters used in this work is listed in Table 1. Elastic effects are quantified by a nondimensional Deborah number, defined here as

$\mathrm{De}=\frac{\lambda U}{R}$

with $U$ representing the bulk velocity in the channel and $R$ the cylinder radius. In the numerical simulations, the inlet
Table 1 Range of the model parameters used in this work

\begin{tabular}{lllll}
\hline Models & $\varepsilon$ & $\beta$ & $\alpha$ & $L^{2}$ \\
\hline UCM & 0 & 0 & 0 & $\infty$ \\
Oldroyd-B & 0 & $0.125 ; 0.4$ and 0.8 & 0 & $\infty$ \\
PTT & 0.02 and 0.25 & 0 & 0 & $\infty$ \\
FENE-CR & 0 & 0.1 & 0 & 10 and 100 \\
Giesekus & 0 & 0.59 & 0.02 & $\infty$ \\
\hline
\end{tabular}

bulk velocity and the cylinder radius were kept constant, therefore the Deborah number was varied by changing the value of the relaxation time. Of course in an experiment with a given fluid and geometry the elasticity number $E=$ $\lambda \eta_{0} / \rho R^{2}$ is kept constant and the Deborah number is varied by changing the flow rate, that is $U$, and consequently the Reynolds number. However, since we force the Reynolds number to be zero and are concerned with steady flows, the only parameter left is $D e$ and it is theoretically irrelevant whether it is $\lambda$ or $U$ that is varied.

All the calculations were carried out with a finite-volume method (for details see Oliveira et al. 1998 and Alves et al. $2000,2001,2003$ ) for the limiting case of $R e=0$, which was imposed numerically by neglecting the convective terms of the momentum equation. Accurate representation of the convective terms in the constitutive equation is of extreme importance in viscoelastic simulations, and the CUBISTA high-resolution scheme developed by Alves et al. (2003) was applied for this purpose. The CUBISTA scheme has the advantage over classical high-resolution schemes (e.g., the SMART scheme by Gaskell and Lau 1988) of promoting better iterative convergence when employed in conjunction with implicit methods. It is a simpler alternative to Lagrangian approaches whose implementation in conjunction with finite element methods is addressed in the review of Baaijens (1998). Additional details of the present method regarding the implementation of the Giesekus model, so that numerical stability is improved, can be found in Oliveira (2001).

\section{Problem description and computational meshes}

A Galilean transformation shows that the settling of a cylinder in a medium confined by two parallel plates is equivalent to the steady uniform flow of a fluid around and past a cylinder in a channel, whose walls move at the same velocity of the approach flow, as shown in Fig. 1. The ratio of channel half-height $h$ to cylinder radius $R$ is set equal to 2 which corresponds to a $50 \%$ blockage case. The computational domain is $80 R$ long, with $19 R$ upstream and $59 R$ downstream of the forward and rear stagnation points of the cylinder, respectively. The downstream length 
Fig. 1 Schematic representation of the flow geometry

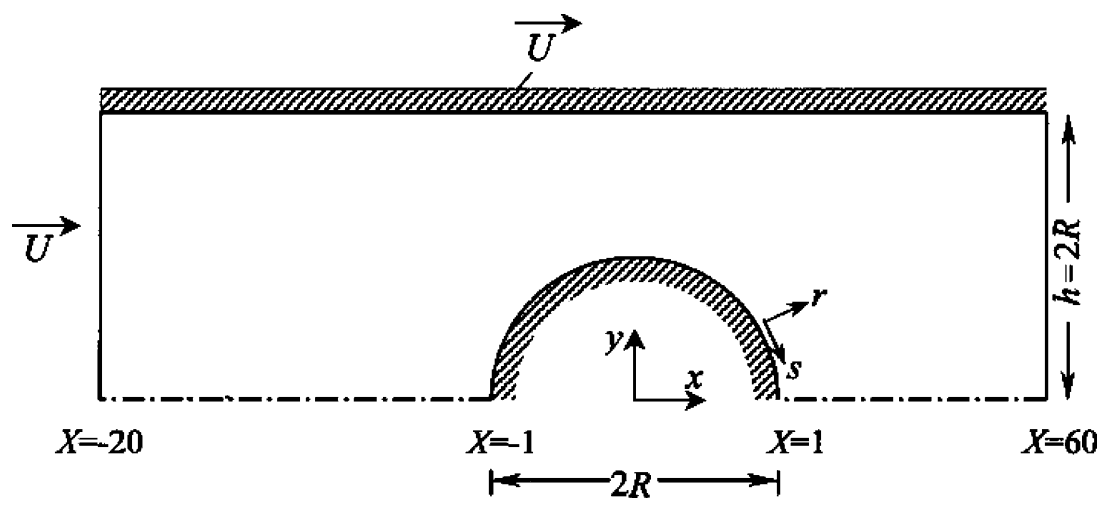

is sufficient for the flow to become fully developed and to avoid any effect of the outflow boundary condition upon the flow in the vicinity of the cylinder. Vanishing axial gradients are applied to all variables, including the pressure gradient, at the outlet plane. No-slip conditions are imposed at both the cylinder surface $(r=R: u=0, v=0)$ and the channel wall $(y=h: u=U)$.

The main characteristics of the meshes used in this work are given in Table 2, including the total number of cells $(\mathrm{NC})$, the number of control volumes around the surface of the cylinder (NS), the number of cells placed radially from the cylinder to the channel wall (NR), and the minimum cell spacing along the radial $(\Delta r)$ and the azimuthal $(\Delta s=r \Delta \theta)$ directions both normalized with the cylinder radius. The first numerical simulations were carried out with mesh M60 of our previous work (for complete details see Alves et al. 2001). Mesh M60 $0_{\mathrm{WR}}$ has the same number of cells in the radial direction as mesh M60, but is more refined along the wake (hence the subscript WR for "wake-refined"). The total number of cells is 22,560 . The high degree of refinement in the rear wake region of the cylinder of mesh $\mathrm{M} 60_{\mathrm{WR}}$ leads to a minimum normalized cell spacing along the azimuthal direction of 0.0006 compared with 0.0157 for mesh M60. Mesh M120 WR was used to check the convergence with mesh refinement at Deborah numbers near the critical value. This mesh has twice the number of cells along both directions as mesh $\mathrm{M} 60_{\mathrm{WR}}$ with $\mathrm{NC}=90,240$ cells. The number of cells on the cylinder surface of mesh M120 WR is 520 , and the minimum normalized cell spacing along the radial and azimuthal directions is 0.002 and 0.0003 , respectively.

\section{Results and discussion}

Results of computations are presented as a scalar integral quantity representative of the flow and as detailed profiles of velocity and stress components in the vicinity of the cylinder. The integral quantity selected was the dimensionless drag coefficient, $C_{\mathrm{D}}$, calculated as:

$C_{\mathrm{D}}=\frac{1}{\eta_{0} U} \int_{\mathrm{S}}\left(\boldsymbol{\tau}_{\mathrm{tot}}-p \mathbf{I}\right) \bullet \mathbf{n} \bullet \mathbf{i} \mathrm{d} \mathbf{S}$

where $\mathbf{I}$ is the unit tensor, $\mathbf{n}$ is the unit vector normal to the cylinder surface and $\mathbf{i}$ is the unitary vector in the $x$-direction (streamwise direction). Stress profiles are shown in the thin stress boundary layer around the cylinder and on the thin high normal stress region downstream the rear wake, to ascertain the quality of the predictions.

For the UCM and Oldroyd-B models, we used two different criteria to assess numerical stability. One method consisted simply in examining the positive definiteness of the conformation tensor, $\mathbf{A}$, that is $\operatorname{det} \mathbf{A}>0$; in addition, Hulsen (1988) demonstrated that for the Oldroyd-B model,

Table 2 Main characteristics of the computational meshes

\begin{tabular}{lllllll}
\hline Mesh & NC & DOF & NR & NS & $(\Delta r / R)_{\min }$ & $(\Delta s / R)_{\min }$ \\
\hline M60 & 17,400 & 104,400 & 60 & 200 & 0.00481 & 0.0157 \\
M60 & 22,560 & 135,360 & 60 & 260 & 0.004 & 0.0006 \\
M120 & 90,240 & 541,440 & 120 & 520 & 0.002 & 0.0003 \\
\hline
\end{tabular}

NC Total number of cells, DOF number of degrees of freedom, NR number of cells placed radially, NS number of cells around the cylinder surface 
one should have det $\mathbf{A} \geq 1$. The conformation and polymer stress tensors are related by

$\boldsymbol{\tau}=\frac{\eta_{\mathrm{P}}}{\lambda}(\mathbf{A}-\mathbf{I})$.

The other criterion for judging the performance of the numerical discretisation is to determine whether the elastic tensor, $\mathbf{T}$,

$\mathbf{T}=\boldsymbol{\tau}+\frac{\eta_{\mathrm{P}}}{\lambda} \mathbf{I}=\frac{\eta_{\mathrm{P}}}{\lambda} \mathbf{A}$,

is positive definite (Dupret and Marchal 1986). Alternatively, the system condition number, $S$, can also be used to indicate a temporal loss of evolution. For a $2 \mathrm{D}$ flow, $S$ is given by (Kim et al. 2005b),

$S=2 \frac{\lambda_{1} \lambda_{2}}{\lambda_{1}^{2}+\lambda_{2}^{2}}=2 \frac{\operatorname{det} \mathbf{T}}{\operatorname{tr}\left(\mathbf{T}^{2}\right)}$,

where $\lambda_{1}$ and $\lambda_{2}$ are the nontrivial eigenvalues of the elastic tensor, T. To guarantee no loss of evolution, $S$ needs to be positive.

In this work, $x$ and $y$ Cartesian coordinates are normalized with the cylinder radius, $R$, the velocity components $(u$ and $v)$ with the characteristic velocity $U$, and the extra stress tensor $\tau$ and the pressure $p$ by $\eta_{0} U / R$.
Mesh refinement studies

It is recognized that this flow problem is difficult to solve accurately because to resolve the very thin stress boundary layer at the cylinder wall and the normal stresses downstream of the rear stagnation point, very refined meshes are required in these regions. The effect of mesh refinement for the UCM model is shown in Fig. 2, where the normalised streamwise normal stresses along the cylinder sidewalls and wake centreline are plotted for $D e=0.6$ and 0.7. In the region of maximum stresses on the cylinder sidewall and the rear wake, there is a small mesh dependency, especially at $D e=0.7$. For comparison, Fig. 2 also presents the $\tau_{\mathrm{xx}}$ profile along the rear wake centreline predicted by Dou and Phan-Thien (2003) with their mesh M4 at $D e=0.6$. A small inaccuracy might result from extracting their data from the original figures; however, their maximum value of $\tau_{x x}$ in the wake still deviates approximately by $9.2 \%$ from our predictions (cf. zoom included in Fig. 2), and such differences are probably related to the insufficient mesh refinement of Dou and Phan-Thien (2003). In fact, their mesh M4 has only a typical $h p$ mesh size of $O\left(10^{-2}\right)$, compared with our mesh size $O\left(10^{-3} ; 10^{-4}\right)$ in mesh M120 $0_{\mathrm{WR}}$. Slight differences present in the results obtained with meshes M60 $0_{\mathrm{WR}}$ and $\mathrm{M} 120_{\mathrm{WR}}$ may be observed in the stress contour plot
Fig. 2 Effect of mesh refinement: stress profiles along cylinder wall and downstream centreplane for UCM fluid. Mesh M60 ${ }_{\mathrm{WR}}$ (dashes); M120 ${ }_{\text {WR }}$ (lines). Symbols (empty circles) from Dou and Phan-Thien (2003) for $D e=0.6$

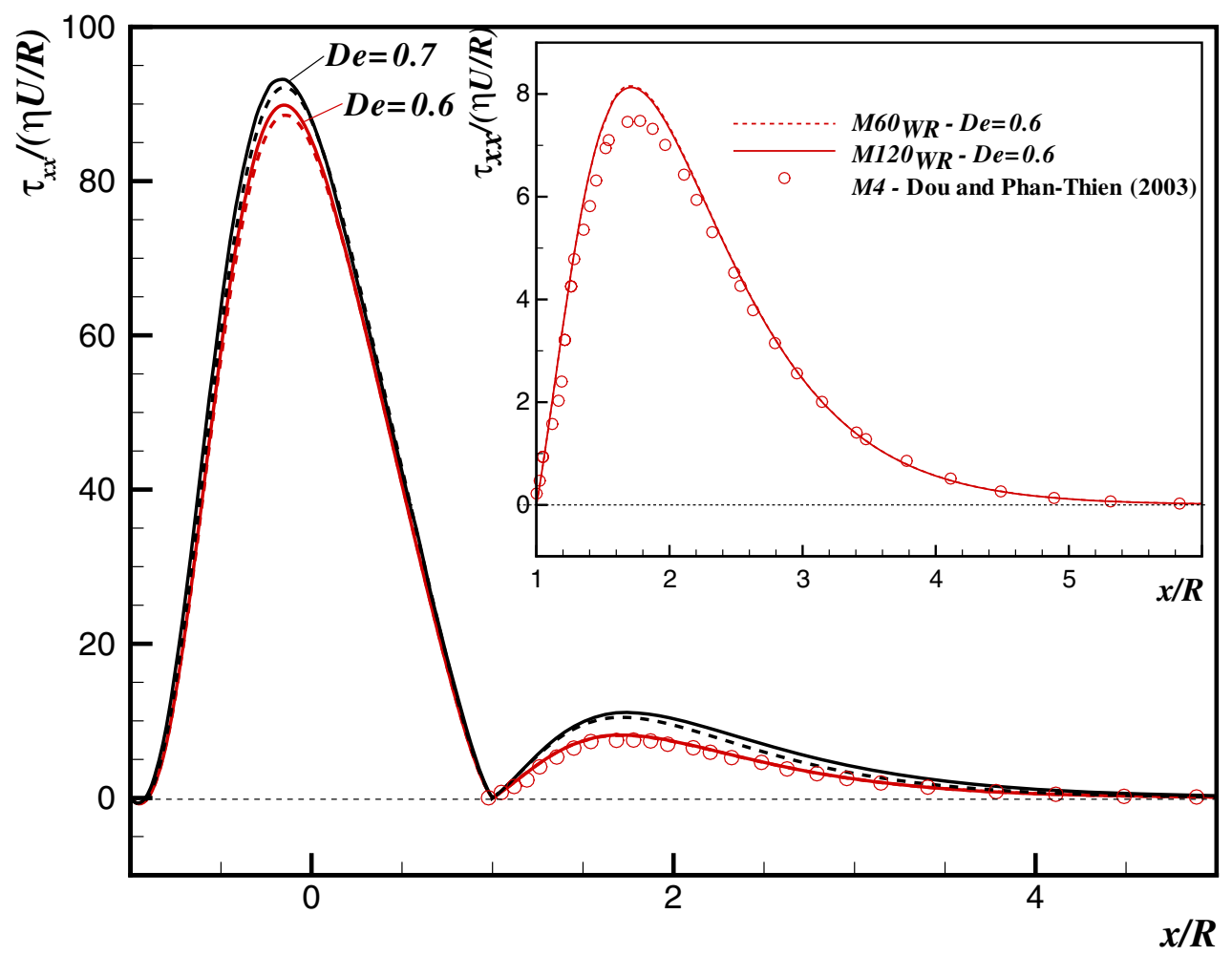


map in Fig. 3, whereas the velocity contours are visually indistinguishable. In contrast to the Newtonian case, the inertialess UCM flow is clearly asymmetric about the $X=0$ plane, particularly the stress fields, with $\tau_{\mathrm{xx}}$ exhibiting maxima along the cylinder sidewall and at the centreline downstream of the cylinder. For the Oldroyd-B model, Fig. 4 shows normal stress profiles along the cylinder sidewalls and centreline under nearly critical conditions. These profiles are for $\beta=0.125(D e=1.0), \beta=0.4(D e=1.1)$ and $\beta=0.2 \quad(D e=1.4)$. Small differences between the solutions on the two meshes are visible near the points of maximum stress, but it should be emphasized that these flow conditions are close to the critical $D e$, when the differences are more discernible. In fact, for lower $D e$, discrepancies in the normal stress profiles, and also in the corresponding peak values, are significantly smaller and even negligible. It has been checked that iteratively converged numerical solutions can still be obtained when $D e$ is increased by 0.1 for the above three cases, but the discrepancies in the predicted peak normal stresses in the wake region with the $\mathrm{M} 60_{\mathrm{WR}}$ and $\mathrm{M} 120_{\mathrm{WR}}$ meshes tended to accentuate. Figure 4 also shows a comparison between our predictions of $\tau_{x x}$ along the rear wake centreline with those of Dou and Phan-Thien (2003) on their mesh M4; a significant difference of the order of $50 \%$ is observed due to the high level of azimuthal refinement of $\mathrm{M} 120_{\mathrm{WR}}$ mesh.

Previous works have shown mesh refinement not to be so crucial with constitutive models exhibiting shearthinning or bounded extensional viscosity (Oliveira et al. 1998, Oliveira 2001, Oliveira and Miranda 2005 and Alves et al. 2000, 2001, 2003), such as FENE-CR, PTT or
Fig. $3 \tau_{x x}$, u and $v$ contour plots for UCM fluid at $D e=0.7$. Mesh $\mathrm{M} 60_{\mathrm{WR}}$ (lines) and $\mathrm{M} 120_{\mathrm{WR}}$ (dashes)

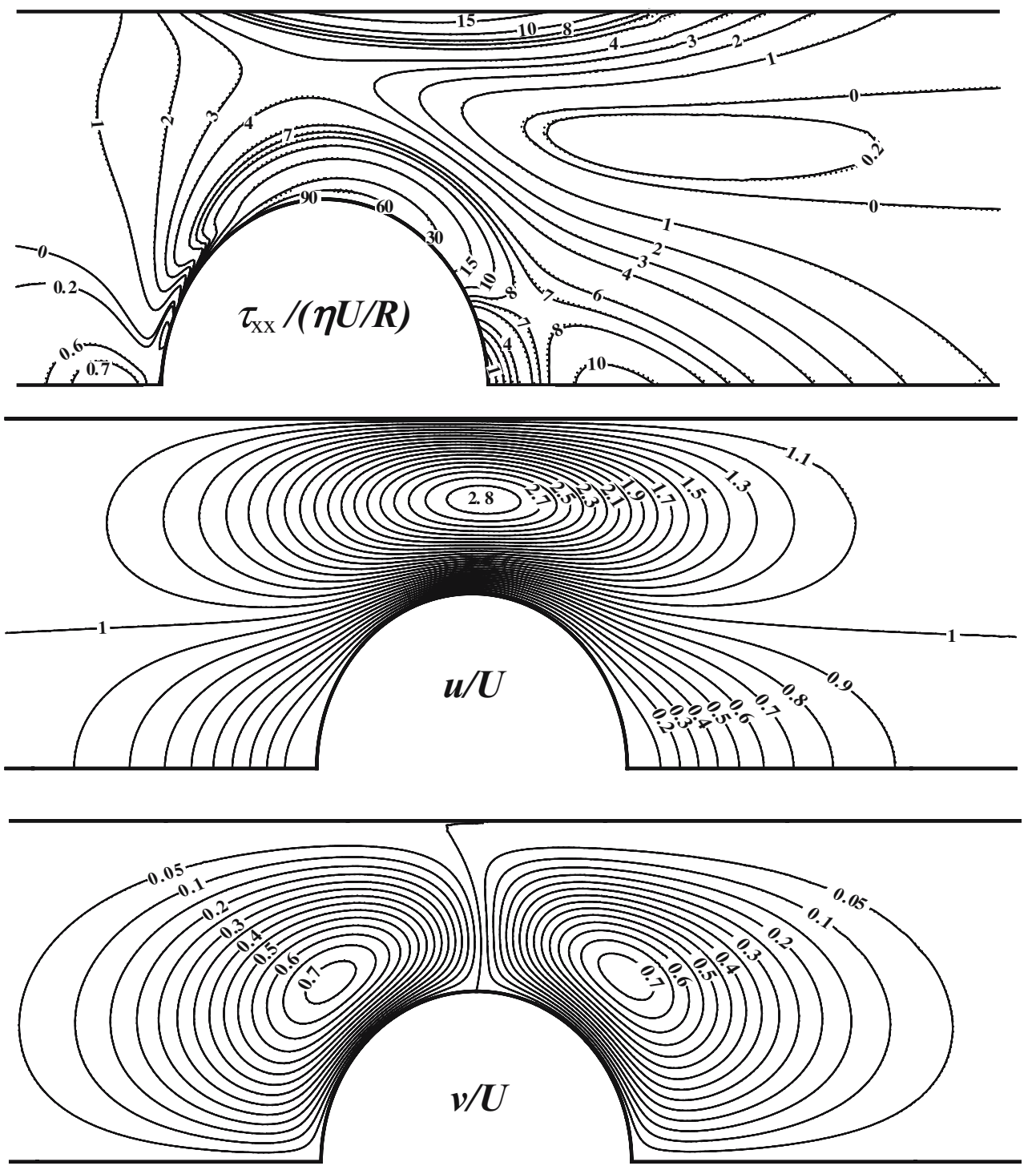


Fig. 4 Effect of mesh refinement: stress profiles along cylinder wall and downstream centreplane for Oldroyd-B fluid. Mesh $\mathrm{M} 0_{\mathrm{WR}}$ (dashed line); $\mathrm{M}^{2} 20_{\mathrm{WR}}$ (full line). Symbols (empty circles) from Dou and Phan-Thien (2003) for $D e=1.0$

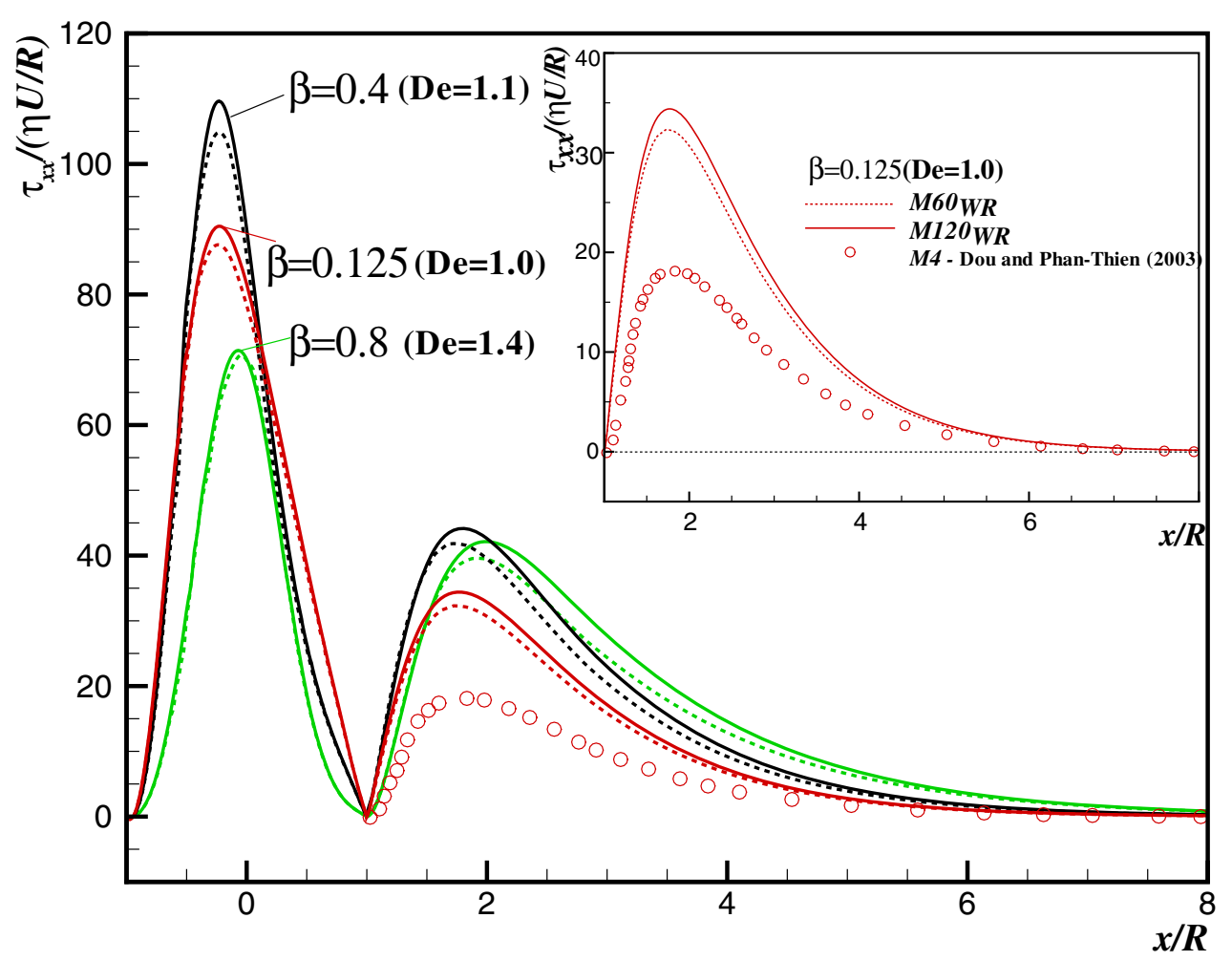

Giesekus models, and therefore, we decided not to use the finer mesh M120 ${ }_{\mathrm{WR}}$ and restrict the simulations with these models to meshes M60 and M60 $\mathrm{WR}$. This decision is corroborated by the comparisons shown in Fig. 5 for the FENE-CR model, where $\tau_{x x}$ predictions are presented near critical Deborah numbers of 5.0 and 4.0 for $L^{2}=10$ and $L^{2}=$ 100, respectively, using meshes $\mathrm{M} 60$ and $\mathrm{M} 60_{\mathrm{WR}}$. Both meshes yield normalized normal stress profiles in good agreement, and also the corresponding peak stresses, an indication of the good accuracy achieved by M60 with the
Fig. 5 Effect of mesh refinement: normal stress profiles along cylinder wall and downstream centreplane for FENECR fluid. Mesh M60 ${ }_{\mathrm{WR}}$ (empty circles) and M60 (lines). Symbols (empty triangles) in the inset are from Kim et al. (2005a)

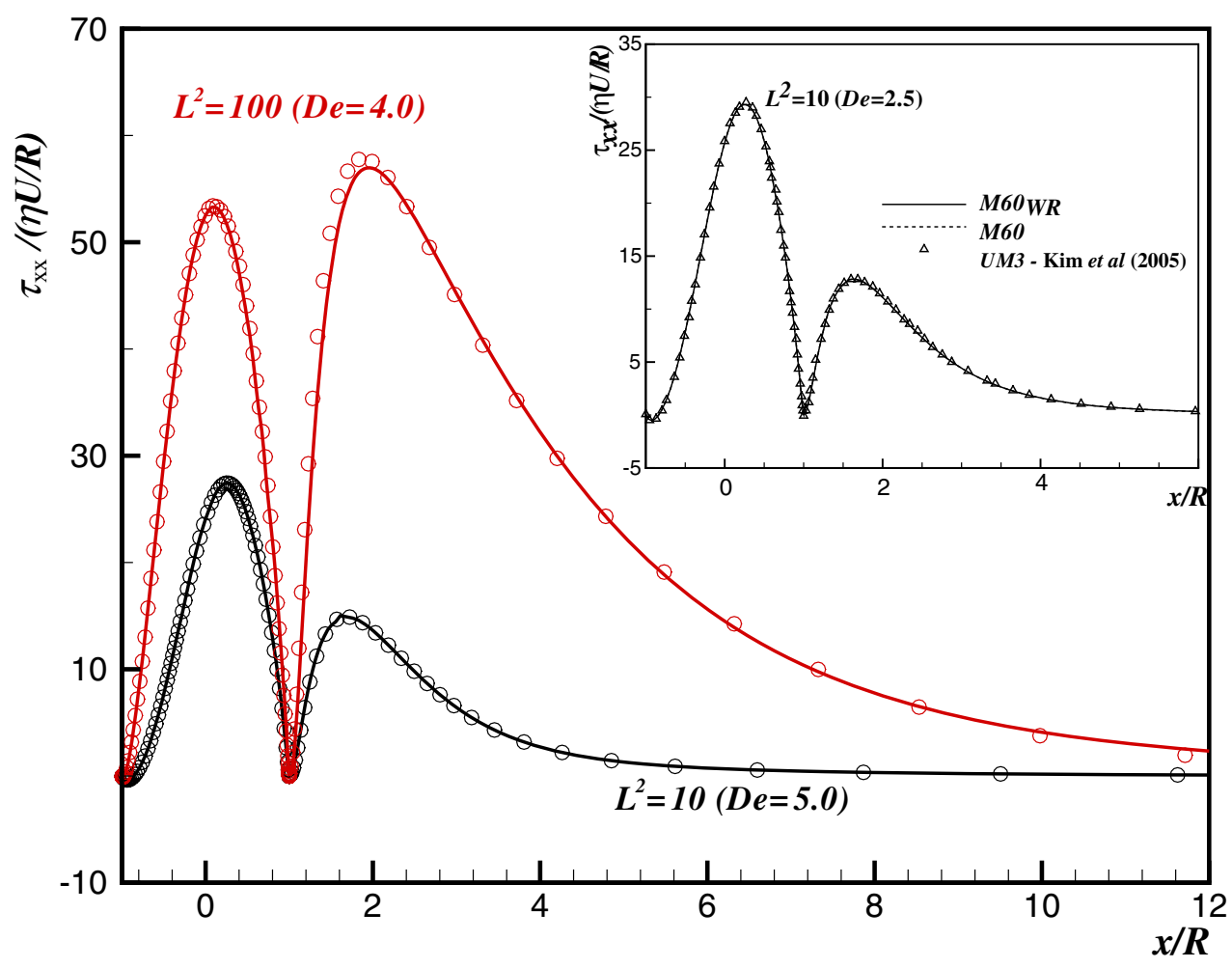




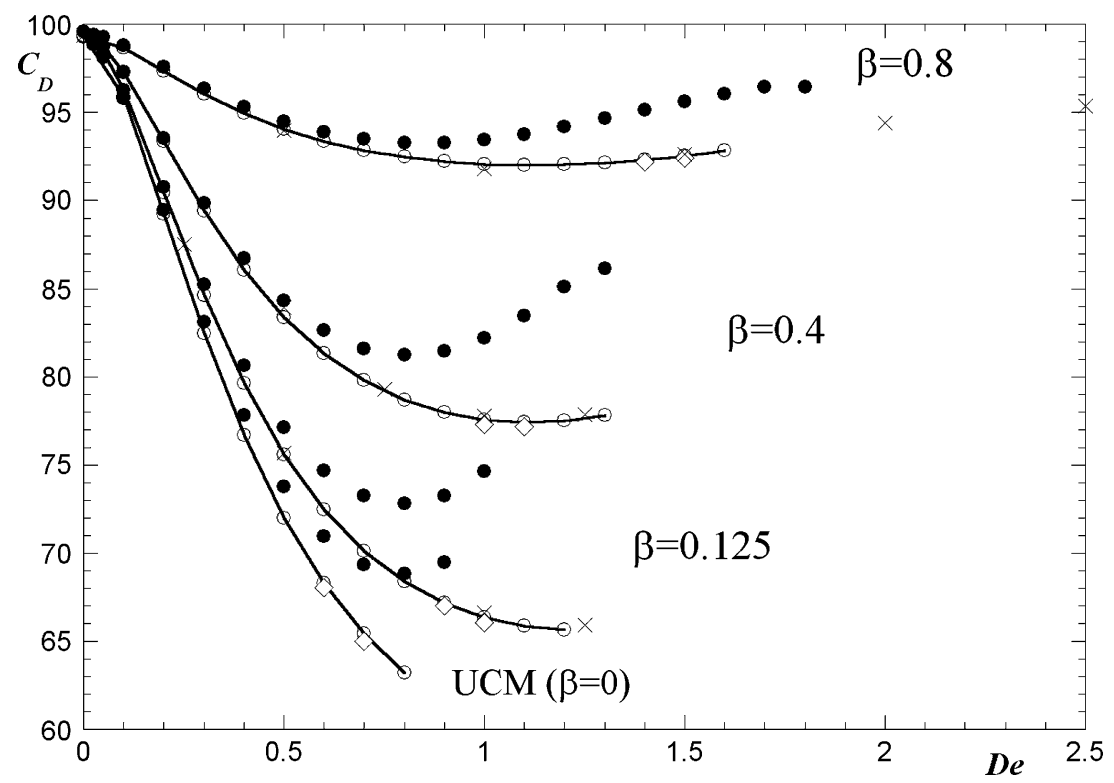

Fig. 6 Drag force coefficient for UCM and Oldroyd-B fluids. Mesh M60 (×); Mesh M60 ${ }_{\mathrm{WR}}(-\mathrm{o}-)$; Mesh M120 $0_{\mathrm{WR}}$ (empty diamonds) and Dou and Phan-Thien (2003) data (filled circles)

FENE-CR model. This mesh independence is also confirmed in the comparison with Kim et al. (2005a) results presented in the inset of Fig. 5. Their predictions at $D e=$ 2.5 with $L^{2}=10$ were obtained with their mesh UM3 having a minimum mesh size of $O\left(10^{-4}\right)$, whereas mesh M60 is only $O\left(10^{-2}\right)$ and yet has similar level of accuracy. Those authors also employed a "high resolution" FEM, and the matching against our data is excellent.
After demonstrating the accuracy of our predictions, in the following sections, we present and discuss separately the results for each constitutive model.

\section{UCM and Oldroyd-B models}

We start with the two simpler quasi-linear differential constitutive models, the upper-convected Maxwell (UCM)

Table 3 Drag force coefficient for the UCM and Oldroyd-B models.

\begin{tabular}{|c|c|c|c|c|c|c|c|c|c|c|c|}
\hline \multirow[t]{2}{*}{$D e$} & \multicolumn{3}{|c|}{ Oldroyd-B $\beta=0.8$} & \multicolumn{3}{|c|}{ Oldroyd-B $\beta=0.4$} & \multicolumn{3}{|c|}{ Oldroyd-B $\beta=0.125$} & \multicolumn{2}{|l|}{ UCM } \\
\hline & M60 & $\mathrm{M} 60_{\mathrm{WR}}$ & $\mathrm{M} 120_{\mathrm{WR}}$ & M60 & M60 ${ }_{\mathrm{WR}}$ & $\mathrm{M} 120_{\mathrm{WR}}$ & M60 & $\mathrm{M} 60_{\mathrm{WR}}$ & $\mathrm{M} 120_{\mathrm{WR}}$ & $\mathrm{M} 60_{\mathrm{WR}}$ & $\mathrm{M} 120_{\mathrm{WR}}$ \\
\hline 0.0 & 99.393 & 99.366 & & 99.393 & 99.366 & & 99.393 & 99.366 & & 99.366 & \\
\hline 0.1 & & 98.653 & & & 97.290 & & & 96.286 & & 95.896 & \\
\hline 0.2 & & 97.354 & & & 93.354 & & & 90.477 & & 89.225 & \\
\hline 0.3 & & 96.059 & & & 89.407 & & & 84.625 & & 82.476 & \\
\hline 0.4 & & 94.955 & & & 86.057 & & & 79.622 & & 76.676 & \\
\hline 0.5 & 93.988 & 94.067 & & 83.960 & 83.384 & & 75.702 & 75.607 & & 71.981 & \\
\hline 0.6 & & 93.378 & & & 81.327 & & & 72.495 & & 68.286 & 68.047 \\
\hline 0.7 & & 92.858 & & & 79.797 & & & 70.142 & & 65.420 & 64.993 \\
\hline 0.8 & & 92.483 & & & 78.707 & & & 68.410 & & 63.242 & \\
\hline 0.9 & & 92.230 & & & 77.989 & & & 67.178 & 66.992 & & \\
\hline 1.0 & 91.807 & 92.081 & & 77.767 & 77.573 & 77.293 & 66.633 & 66.354 & 66.045 & & \\
\hline 1.1 & & 92.024 & & & 77.429 & 77.187 & 65.925 & 65.860 & & & \\
\hline 1.2 & & 92.049 & & & 77.508 & & & 65.654 & & & \\
\hline 1.3 & & 92.149 & & & 77.885 & & & & & & \\
\hline 1.4 & & 92.317 & 92.207 & & & & & & & & \\
\hline 1.5 & 92.633 & 92.541 & & & & & & & & & \\
\hline 1.6 & & 92.842 & & & & & & & & & \\
\hline 2.0 & 94.425 & & & & & & & & & & \\
\hline 2.5 & 95.953 & & & & & & & & & & \\
\hline
\end{tabular}


and Oldroyd-B models. Evidence from the literature on viscoelastic flow past a confined cylinder shows that an increase in viscoelasticity tends to reduce the drag force. As shown in Fig. 6 and Table 3, this tendency is replicated by our predictions for the case of sedimentation of a cylinder in a viscoelastic fluid medium obeying the UCM and Oldroyd-B models. Figure 6 also compares the $C_{\mathrm{D}}$ values obtained using meshes $\mathrm{M} 60_{\mathrm{WR}}$ and $\mathrm{M} 120_{\mathrm{WR}}$ with those of Dou and Phan-Thien (2003) obtained with a CVFEM method. All sets of data show a decrease in $C_{\mathrm{D}}$ with Deborah number, but in contrast to the present data, the drag coefficient predicted by Dou and Phan-Thien (2003) always show an increase at high $D e$ numbers, whereas our data only exhibits this increase for large values of $\beta$. Another important difference is that for all retardation ratios, the results are well below the predictions of Dou and Phan-Thien (2003), with agreement only in the range Des 0.3 . As higher values of drag coefficient are usually associated with coarse meshes (Alves et al. 2001) or insufficient accuracy, the discrepancies in Fig. 6 are an indication of loss of accuracy in the results of Dou and Phan-Thien (2003). With the UCM model, stable and iteratively converged simulations could be obtained up to $D e=0.85$ with mesh $\mathrm{M} 60_{\mathrm{WR}}$ and $D e=0.7$ with mesh $\mathrm{M} 120_{\mathrm{WR}}$. For $D e=0.85$ and mesh $\mathrm{M} 60_{\mathrm{WR}}$, a periodic solution leading to oscillating drag force values was observed.

Figure 7 presents the two stability factors discussed above, the system condition number and the determinant of the conformation tensor, along the cylinder surface and the downstream centreline for a range of Deborah numbers. For all simulations, the minimum values of $S$ and $\operatorname{det} \mathbf{A}$ are always positive, showing no loss of evolution when the flow is steady. It can also be observed that the determinant of the conformation tensor increases with Deborah number both on the cylinder sidewall and in the rear wake zone, indicating strong normal stress effects (in shear and extension, respectively), while $S$ behaves inversely (the two quantities are seen to have symmetric shapes relative to $S=$ det $\mathbf{A}=1$ line when plotted in a log-scale). In the rear stagnation point, both system condition numbers $S$ and det A tend to unity. The results in Fig. 7 suggest $S$ as being a better indication of loss of evolution.

Figure 8 presents normal stress and velocity profiles along the cylinder surface and the centreline downstream of the cylinder as function of Deborah number for the UCM model. The $\tau_{x x}$ profiles in Fig. 8a show increasing stresses and peak values with Deborah number, with the maximum at the cylinder sidewall larger than the peak at the wake. Near the critical Deborah number $(D e=0.8)$, the wake maximum normal stress is only $20 \%$ of the larger normal stress at the cylinder wall, in contrast to the related problem with stationary channel walls where the wake normal stress maxima attains similar values to the cylinder peak.

According to Oliveira et al. (1998), Alves et al. (2001) and Dou and Phan-Thien (2003, 2004), there is no "negative wake" with UCM fluids for both Poiseuille and uniform approach flows. This is confirmed here for uniform flow in Fig. 8b, where no velocity overshoots in the wake
Fig. 7 Stability factors $[S$ factor and $\operatorname{det}(\mathbf{A})]$ on the centreline for UCM fluid and increasing Deborah numbers $(D e=0.2,0.4$, $0.6,0.7$ and 0.8 )

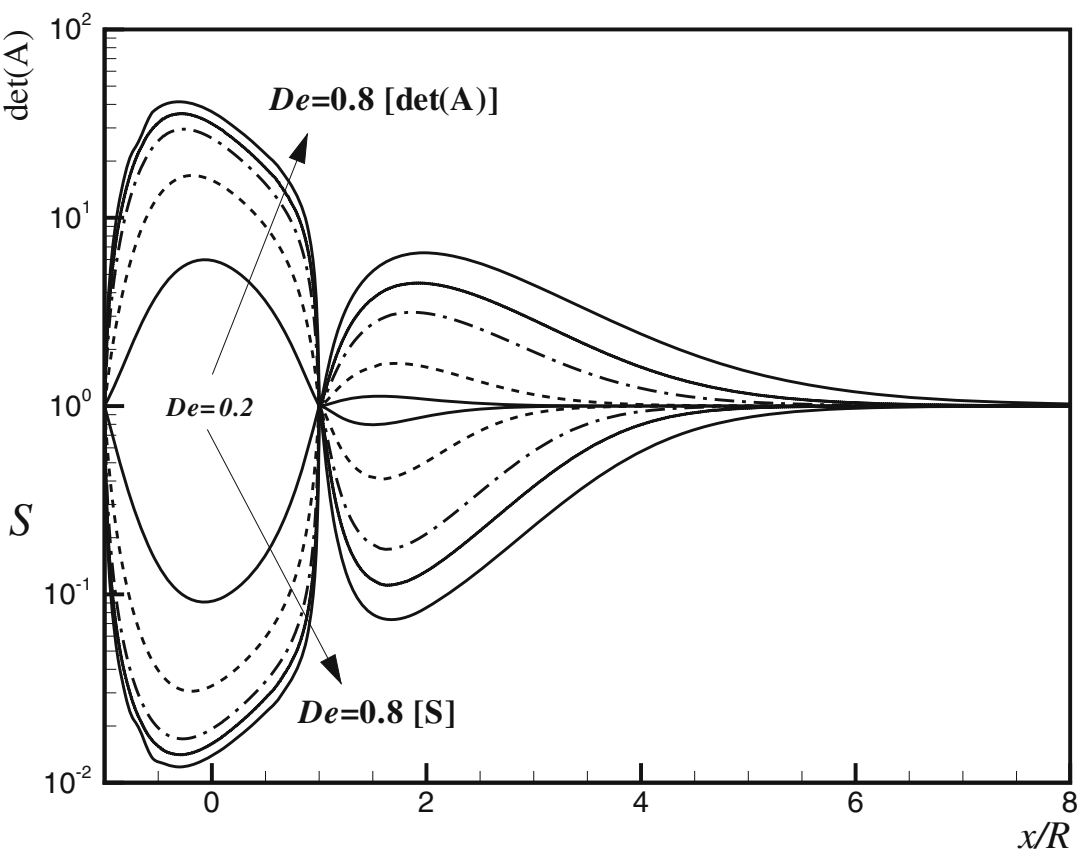


Fig. 8 a $\tau_{x x}$ and $\mathbf{b} u$ centreline profiles for UCM fluid. The inset shows the wake velocity difference, $\Delta u / U=\left(u-u_{\text {New }}\right) / U$ a

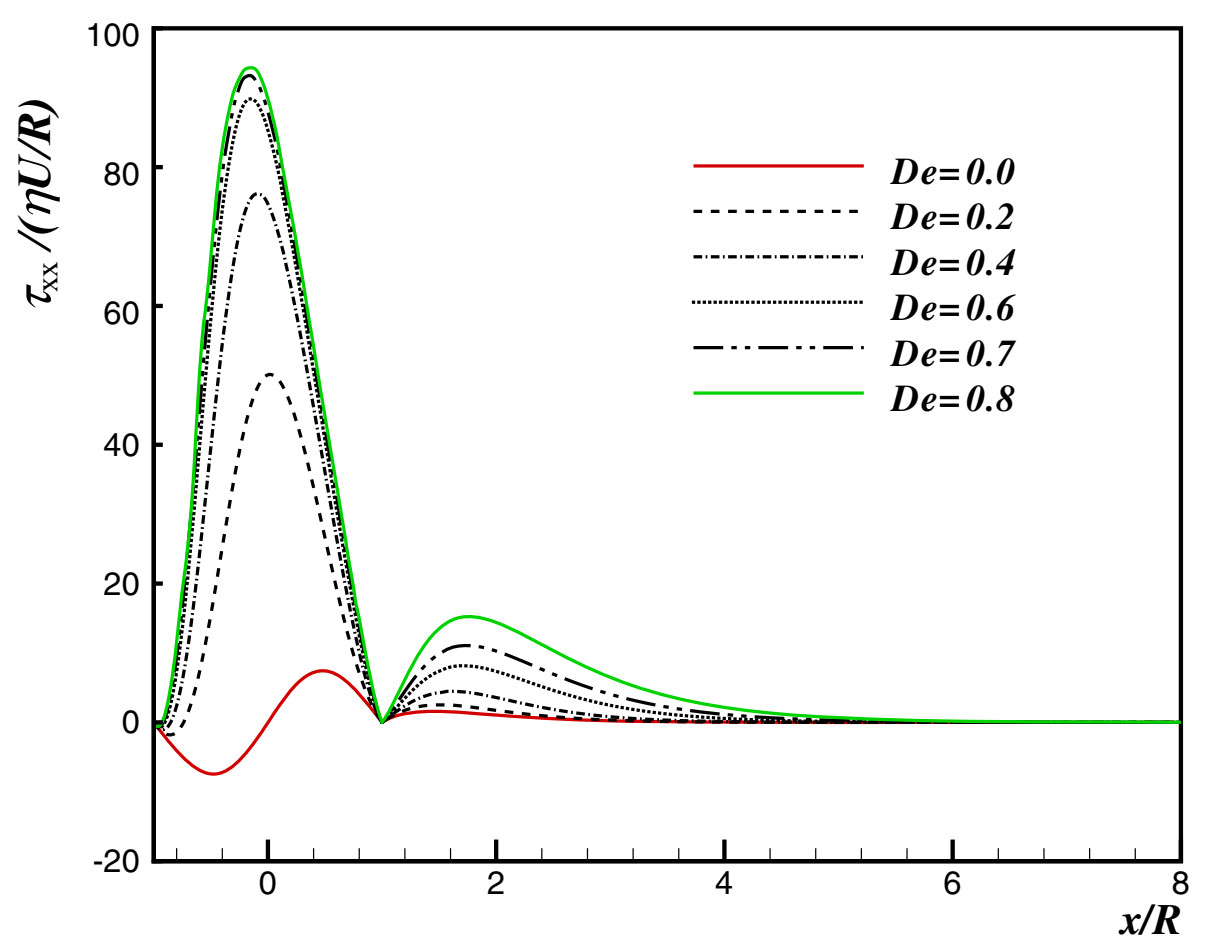

b

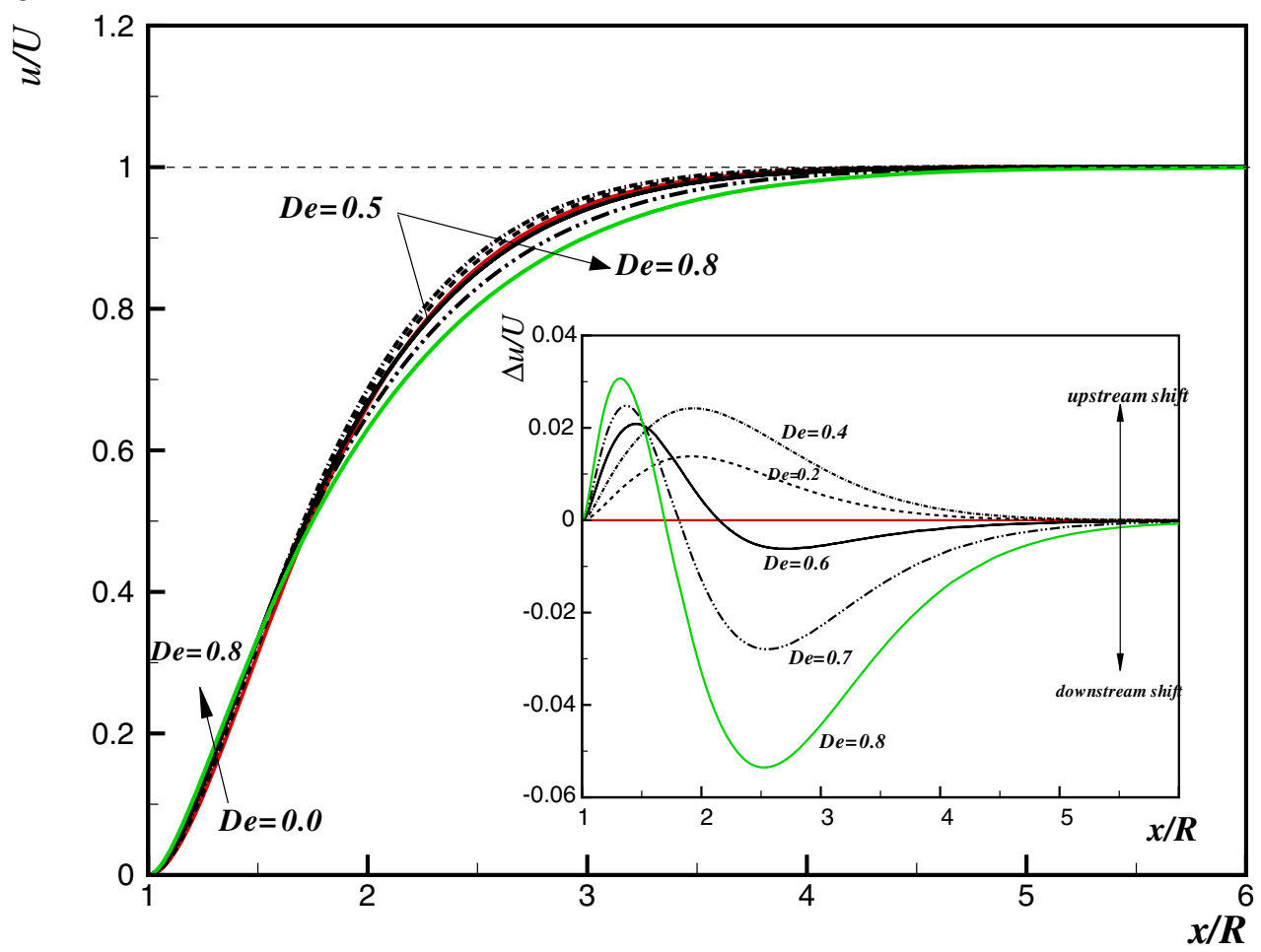

are observed. A small global upstream shift in the velocity profiles with respect to the Newtonian case is seen up to $D e \approx 0.5$ (the inset shows the difference, $\Delta u=u-u_{\text {Newtonian}}$ ). Then, for $D e>0.5$ the behaviour observed becomes more complex with the velocity profiles exhibiting both an upstream shift near the rear stagnation point followed by a downstream shift further downstream of the cylinder. Similar observations of a downstream shift in the elastic wake behind a cylinder in a channel were reported by McKinley et al. (1993). This phenomenon has been found to increase monotonically with $D e$ in all experimental studies to date. 
For the Oldroyd-B model, the results are globally similar to those obtained with the UCM model, with slight differences discussed below. Using three different viscosity ratio values $(\beta=0.125, \beta=0.4$ and $\beta=0.8)$, stable and converged simulations on mesh $\mathrm{M} 60_{\mathrm{WR}}$ could be obtained up to $D e=1.2, D e=1.3$ and $D e=1.6$, respectively. In terms of the normalized velocity components and the normal stress contour maps for these cases, the behaviour follows closely Fig. 3 for the UCM, and the plots are therefore not shown here for conciseness. An important difference
Fig. 9 a $\tau_{x x}$ and $\mathbf{b} u$ centreline profiles for the Oldroyd-B fluid with $\beta=0.125$. The inset shows the wake velocity difference, $\Delta u / U=\left(u-u_{\text {Newt }}\right) / U$ a

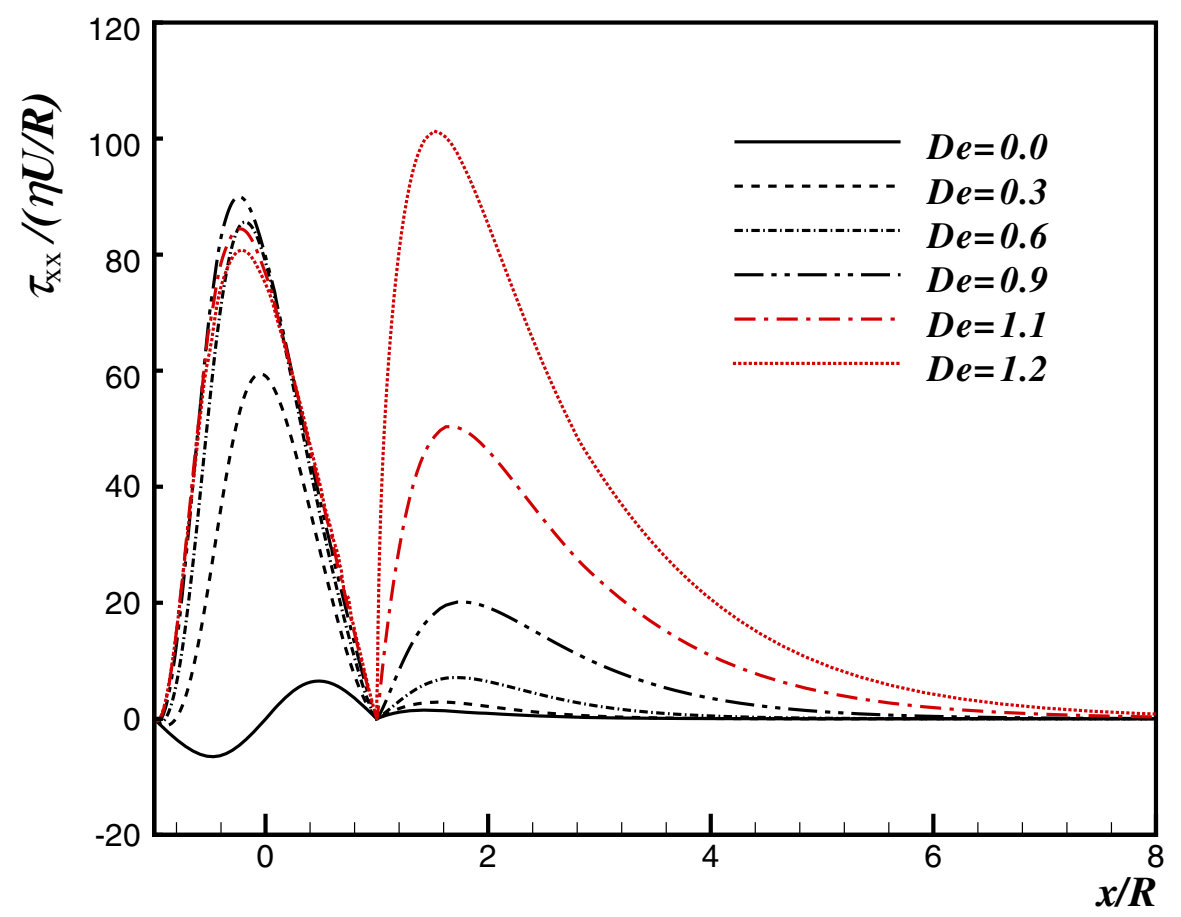

b

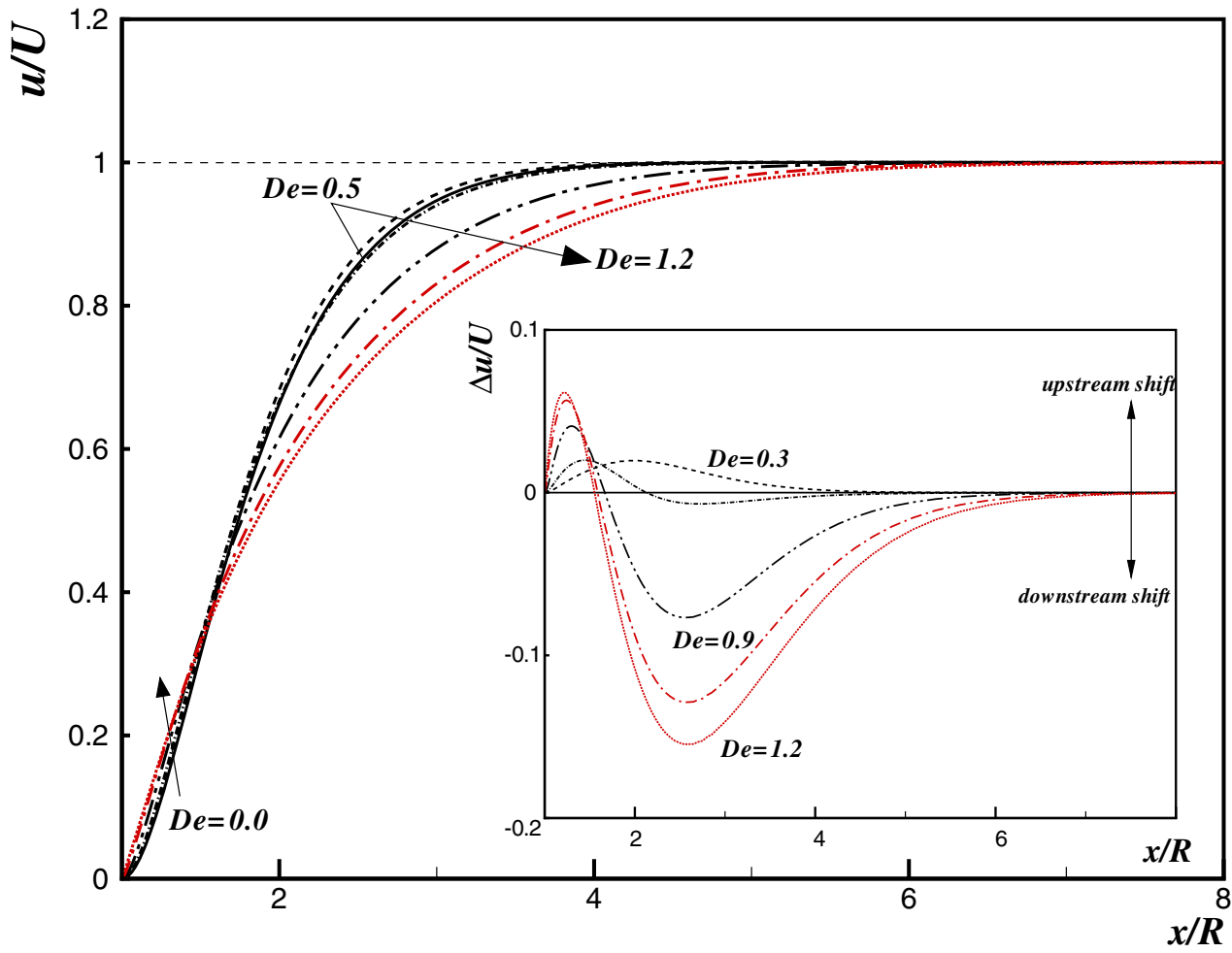


between Oldroyd-B and UCM fluids can be observed by comparing the normal stress profiles for $\beta=0.125$ in Fig. 9a with those for $\beta=0$ in Fig. 8a. At low elasticity and as for the UCM model, the normal stress on the cylinder sidewall increases significantly with Deborah number up to $D e=0.9$; however, above $D e=1.0$, there is a change of trend for the
Oldroyd-B fluid, and $\tau_{x x}$ starts decreasing with $D e$. The behaviour in the rear wake zone is also noteworthy: for the Oldroyd-B model, the normal stress increases strongly with $D e$, and near the critical value, the maximum value of $\tau_{x x}$ in the rear wake is higher than in the cylinder sidewall, whereas for the UCM model, the rear peak values are well
Fig. 10 a $\tau_{x x}$ and $\mathbf{b} u$ centreline profiles for the Oldroyd-B fluid with $\beta=0.4$ and $\beta=0.8$. The inset shows the wake velocity difference, $\Delta u / U=\left(u-u_{\text {Newt }}\right) / U$ a

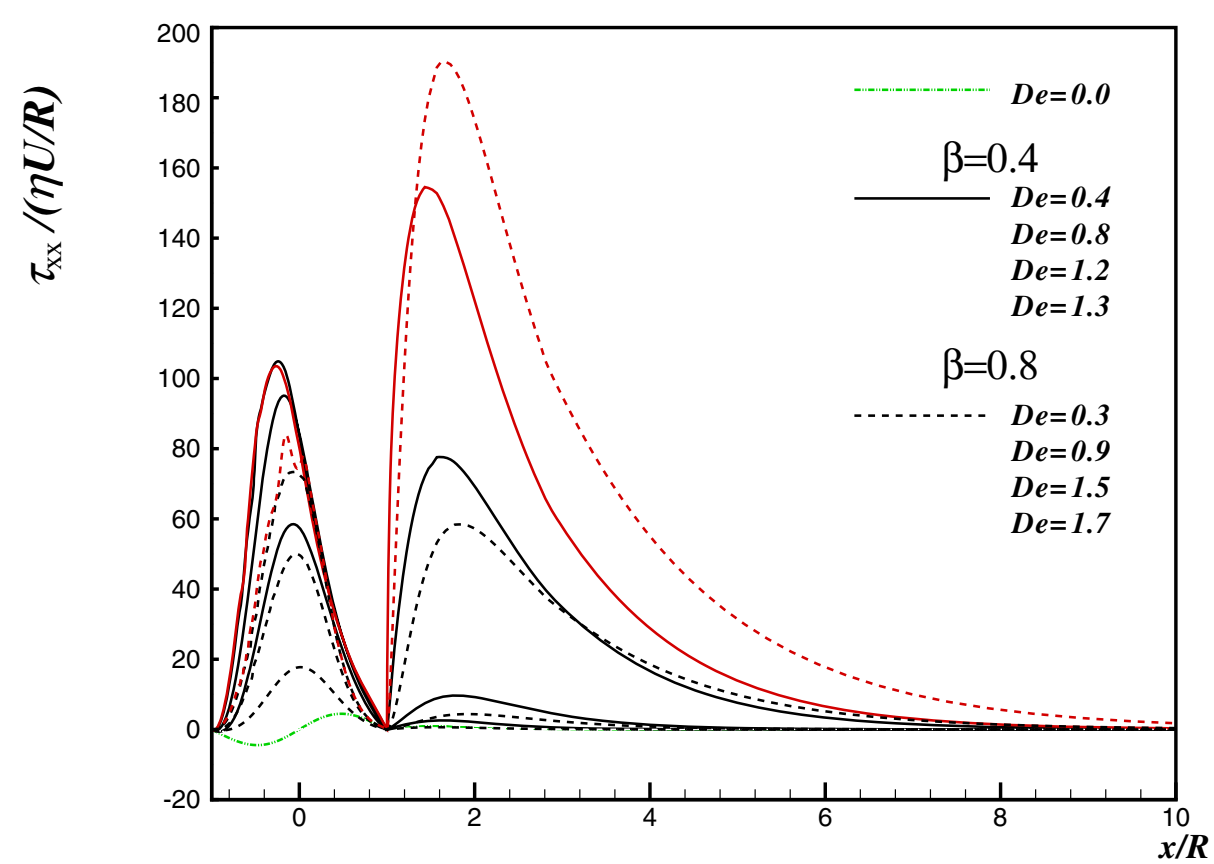

b

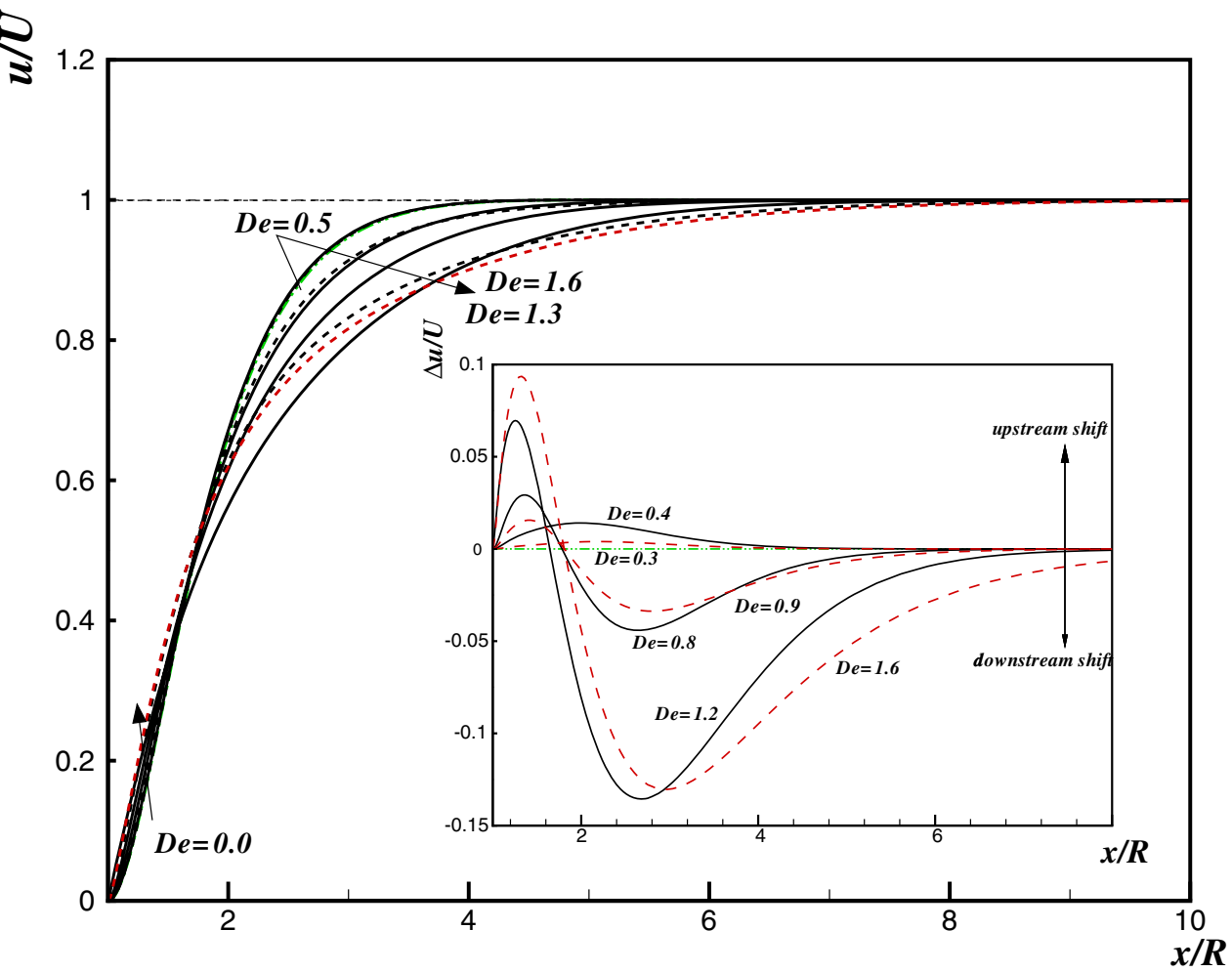


Fig. 11 Drag force coefficient for the FENE-CR fluid. Mesh M60 ${ }_{\mathrm{WR}}(-0-)$; Mesh M60 (×); Kim et al. (2005a) data (filled diamonds)

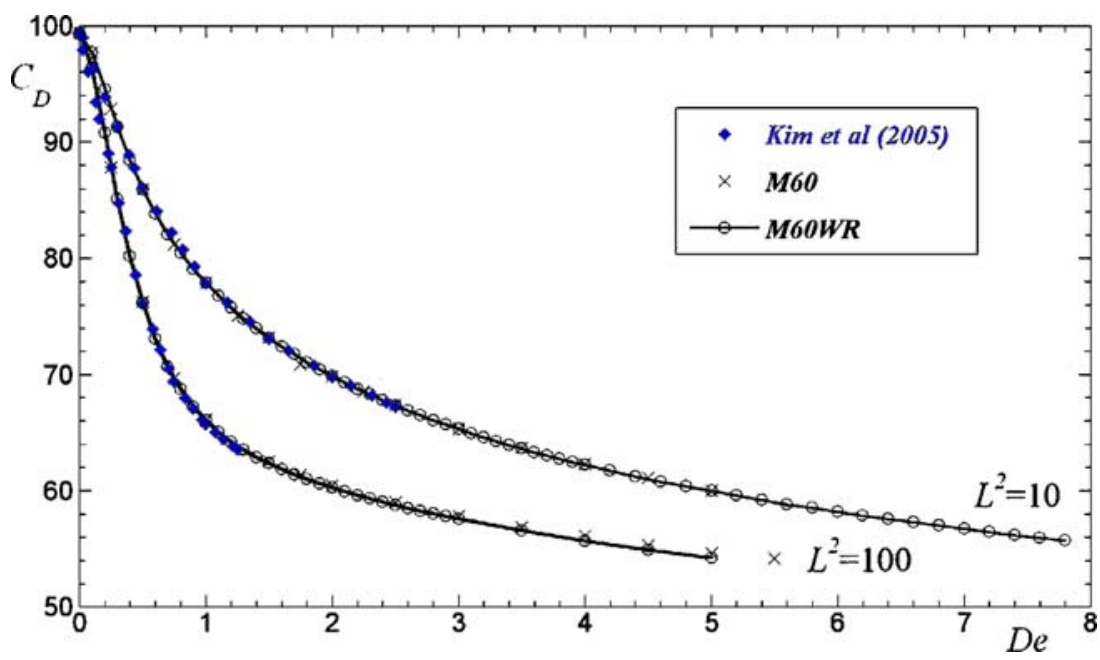

below the cylinder peak values. This feature occurs for all models except for the UCM fluid, probably because for this model, we cannot obtain steady results for $D e$ above unity.

The velocity profiles presented in Fig. 9b illustrate the absence of negative wake for the Oldroyd-B model and show the same trends seen previously with the UCM model, i.e., an upstream shift at all $D e$ near the rear stagnation point followed by a downstream shift for $D e \geq$ 0.5 . The axial position where the transition from upstream to downstream shift occurs decreases with Deborah number from $x / R \approx 2.2$ at $D e=0.6$ to $x / R \approx 1.6$ at $D e=1.2$.

When decreasing polymer concentration of the Oldroyd-B model (increasing the viscosity ratio factor to $\beta=0.4$ and $\beta=$ 0.8 ), the global behaviour for all variables remains qualitatively similar to that of the $\beta=0.125$ case discussed above. Figure $10 \mathrm{a}$ shows the normalized normal stress profiles at high Deborah numbers $(D e=1.2,1.3$ for $\beta=0.4$ and $D e=1.5$, 1.7 for $\beta=0.8)$. The peak values on the cylinder sidewalls saturate and then decrease, whereas in the wake, the second peak exceeds the first at high $D e$. As the critical $D e$ has increased with the increase of $\beta$, the peaks values of $\tau_{x x}$ in the wake, due to viscoelastic extensional effects, can rise to higher values than before (smaller $\beta$ ). Regarding the profile at $D e=1.7$ and $\beta=0.8$ in Fig. 10a, although some oscillations are visible on the cylinder wall zone and in the total drag force, the corresponding simulation did not show signs of iterative divergence, presenting positive values of $\min (S)$ and $\min (\operatorname{det} \mathbf{A})$. These results are not shown here for conciseness; anyway, the waviness of $\tau_{x x}$ is most certainly the first indication that the maximum allowable $D e$ is about to be reached.

\section{FENE-CR Model}

A slightly modified version of the FENE-CR model was proposed and used by Coates et al. (1992) and later by a number of other authors in a variety of studies, such as in cylinder and sphere problems, both with uniform and Poiseuille approach flow conditions (McKinley et al. 1993, Kim et al. 2005a, Oliveira and Miranda 2005, Satrape and Crochet 1994, Harlen 2002 and Dou and Phan-Thien 2004). In all these works, the constant viscosity FENE-CR model predicted the onset of negative wake in the velocity profiles for sufficiently low levels of the extensibility parameter. In the present investigation, the FENE-CR model was applied with $\beta=0.1$ and two extensibility values, $L^{2}=10$ and $L^{2}=100$, to assess the influence of this model parameter on the negative wake phenomenon.

Table 4 Drag force coefficient for the FENE-CR model

\begin{tabular}{|c|c|c|c|c|}
\hline \multirow[t]{2}{*}{$D e$} & \multicolumn{2}{|c|}{ FENE-CR $L^{2}=10$} & \multicolumn{2}{|c|}{ FENE-CR $L^{2}=100$} \\
\hline & M60 & $\mathrm{M} 60_{\mathrm{WR}}$ & M60 & $\mathrm{M} 60_{\mathrm{WR}}$ \\
\hline 0.1 & 97.715 & 97.709 & & 96.426 \\
\hline 0.2 & & 94.575 & & 90.775 \\
\hline 0.3 & & 91.308 & & 85.072 \\
\hline 0.4 & & 88.419 & & 80.178 \\
\hline 0.5 & 85.988 & 85.935 & 76.263 & 76.218 \\
\hline 1.0 & 77.902 & 77.881 & 66.144 & 66.037 \\
\hline 1.5 & 73.188 & 73.156 & 62.502 & 62.343 \\
\hline 2.0 & 69.875 & 69.862 & 60.458 & 60.261 \\
\hline 2.5 & 67.380 & 67.329 & 59.009 & 58.769 \\
\hline 3.0 & 65.341 & 65.306 & 57.839 & 57.578 \\
\hline 3.5 & 63.679 & 63.629 & 56.846 & 56.618 \\
\hline 4.0 & 62.273 & 62.222 & 56.119 & 55.796 \\
\hline 5.0 & 60.017 & 59.968 & 54.612 & 55.069 \\
\hline 6.0 & & 58.199 & & \\
\hline 7.0 & & 56.764 & & \\
\hline 7.8 & & 55.716 & & \\
\hline
\end{tabular}


Fig. $12 \tau_{x x}, u$ contour plots for FENE-CR with a $L^{2}=100$ and b $L^{2}=10$ fluid at $D e=3.0$ on mesh M60 $0_{\mathrm{WR}}$

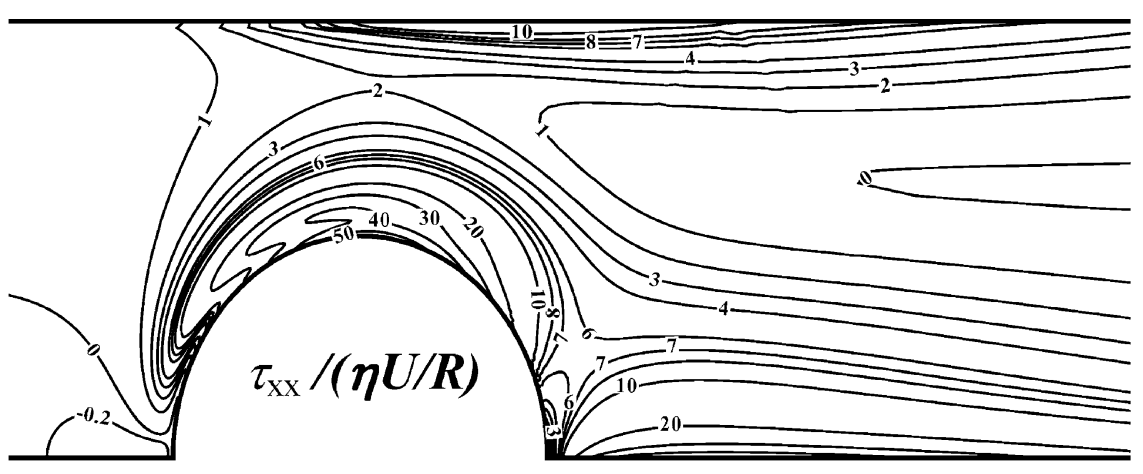

a
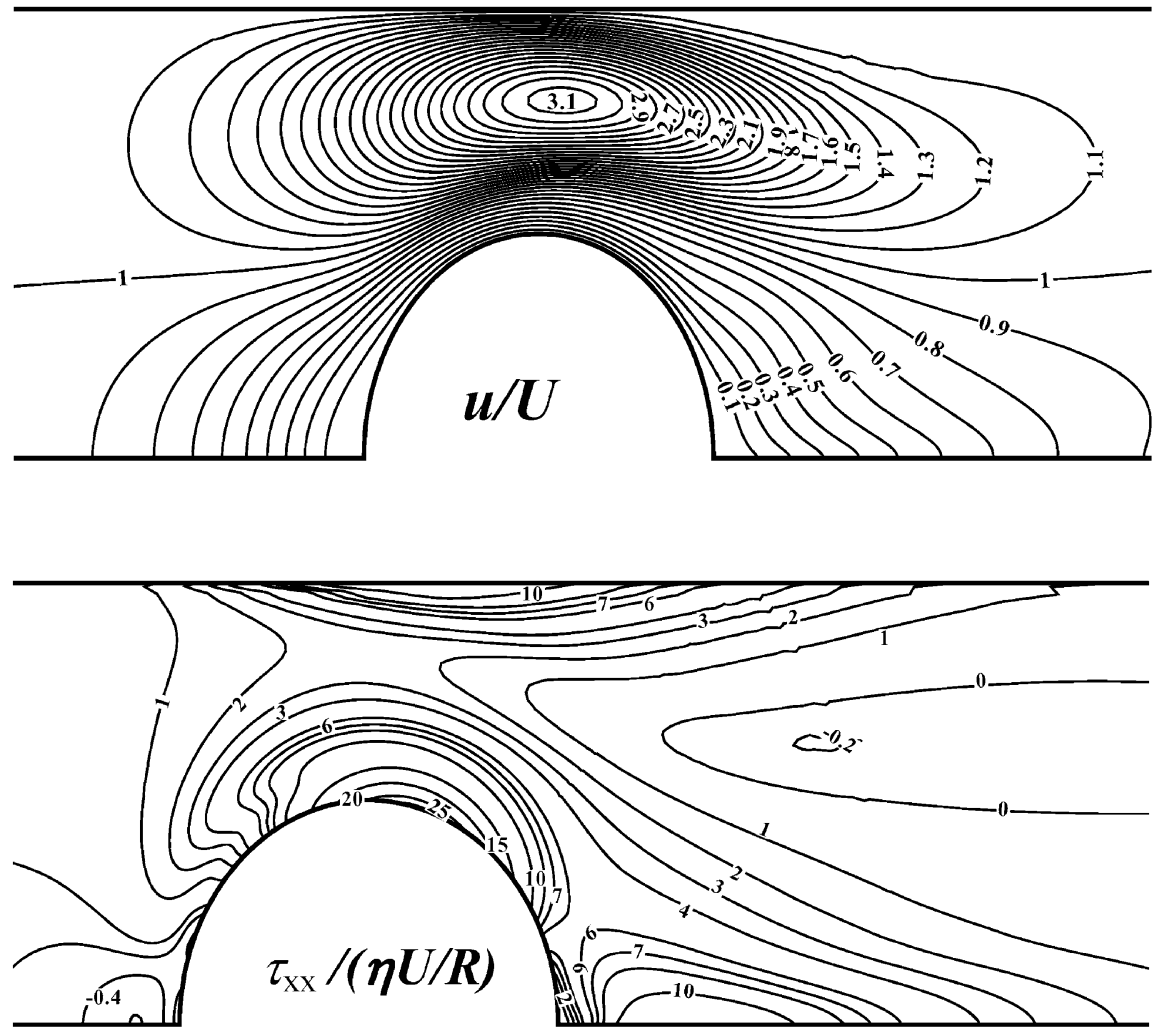

b

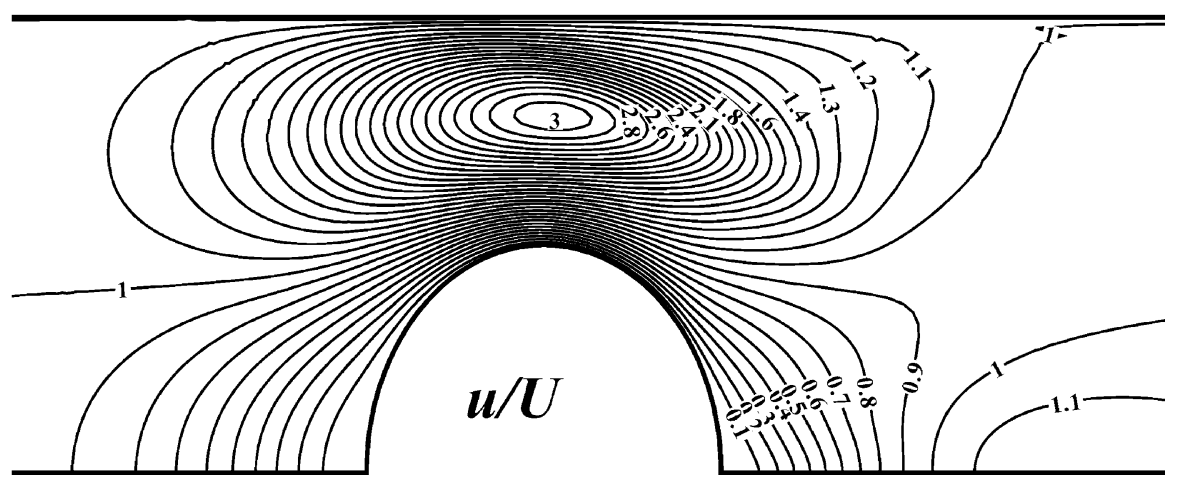


Figure 11 compares the values of $C_{\mathrm{D}}$ obtained in this work with those of Kim et al. (2005a). Our simulations were carried out on meshes M60 and M60 ${ }_{\mathrm{WR}}$; the data are given in Table 4, and it was found that the drag force coefficient decreases monotonically with the Deborah number. Iterative convergence was possible up to $D e=7.8$ and $D e=5.0$ for $L^{2}=10$ and $L^{2}=100$, respectively. Remarkably good agreement was found with the recent results of
Fig. $13 u$ centreline profiles for FENE-CR a $L^{2}=100$ and $\mathbf{b} L^{2}=$ 10 fluids. The inset shows the wake velocity difference, $\Delta u / U=\left(u-u_{\text {Newt }}\right) / U$ a

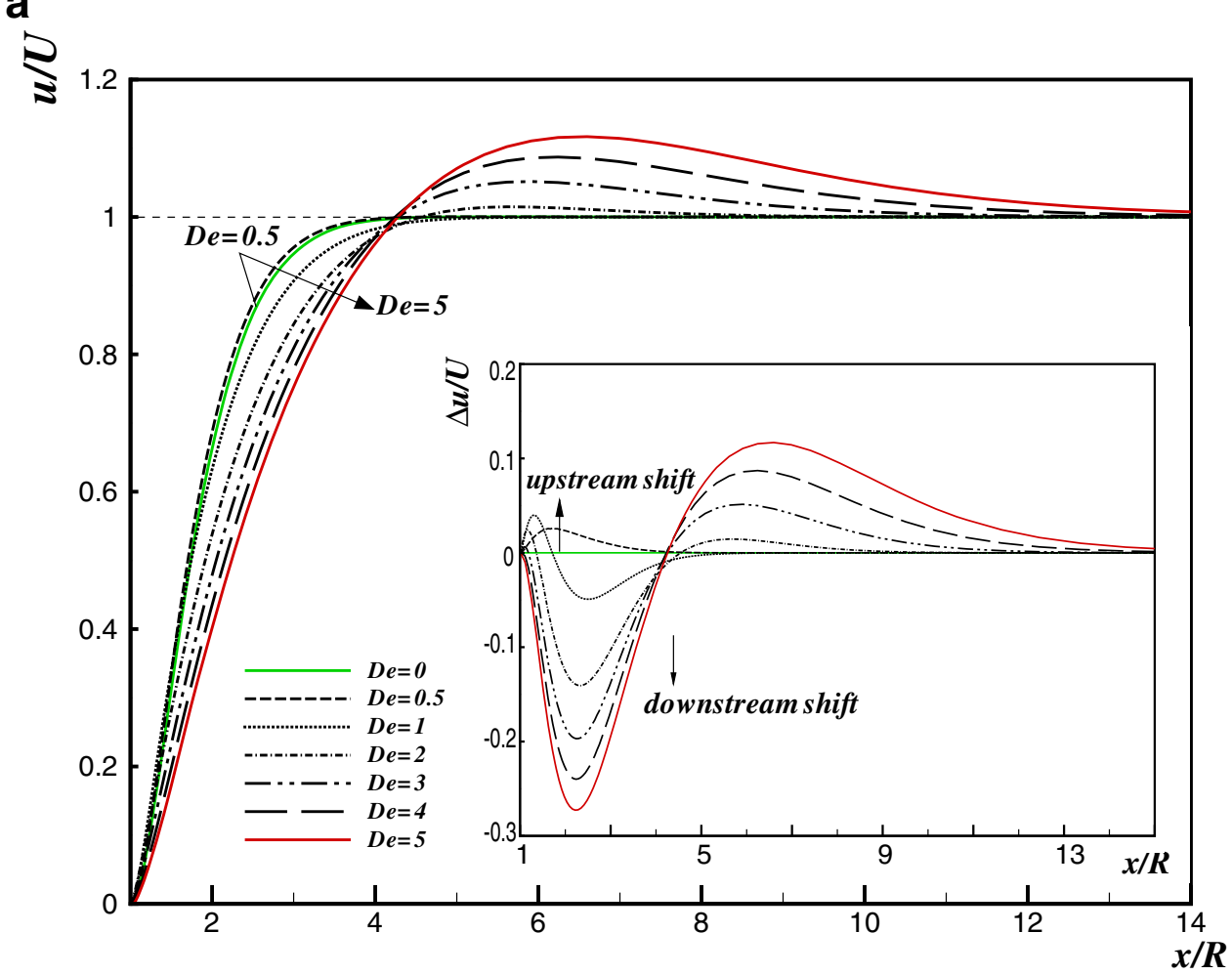

b

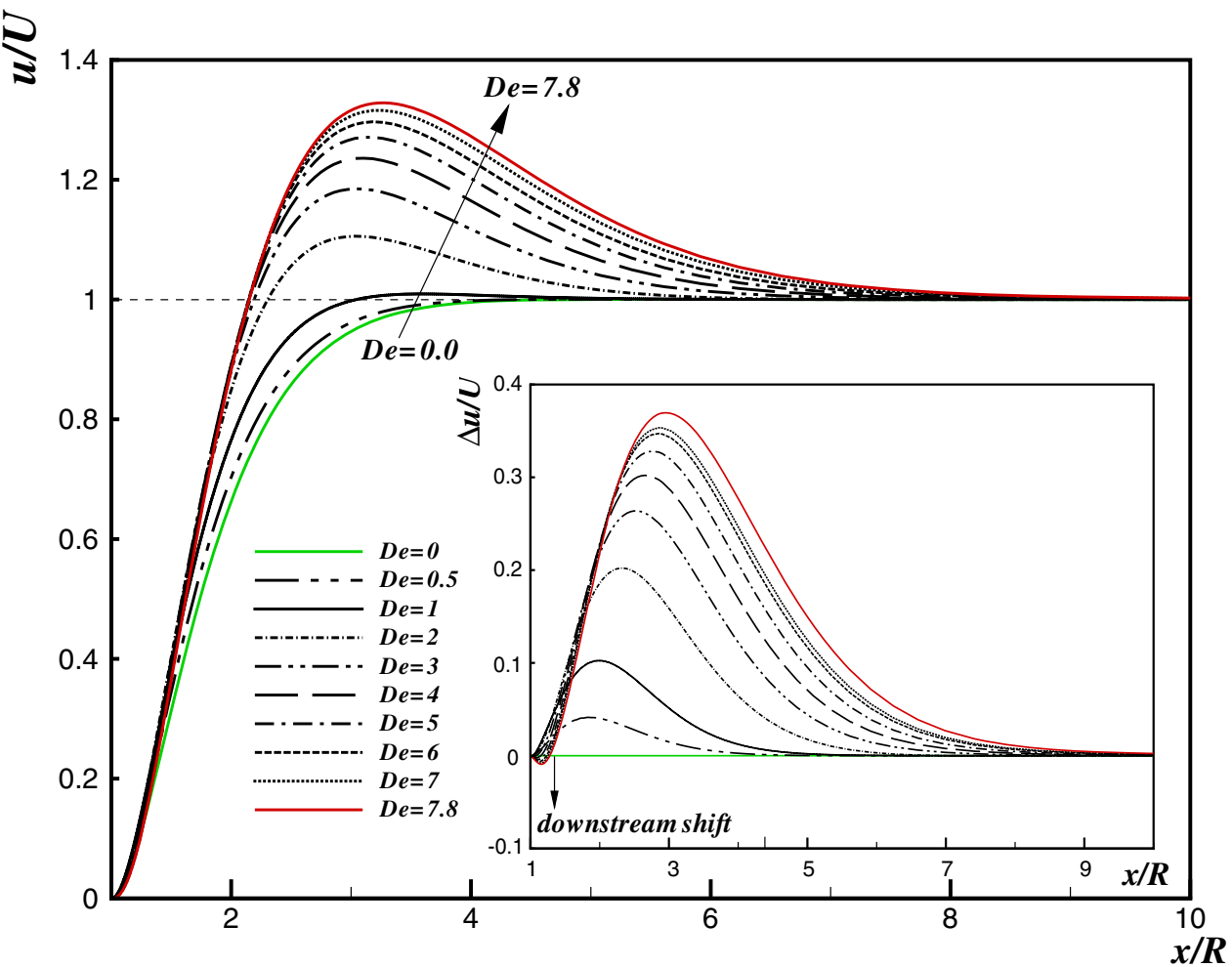


Fig. 14 Drag force coefficient for a PTT fluid with $\beta=0$. Mesh M60 ${ }_{\mathrm{WR}}$ (-o- or -口-); Mesh M60 $(\times)$; and Dou and Phan-Thien (2003) data (filled circles)

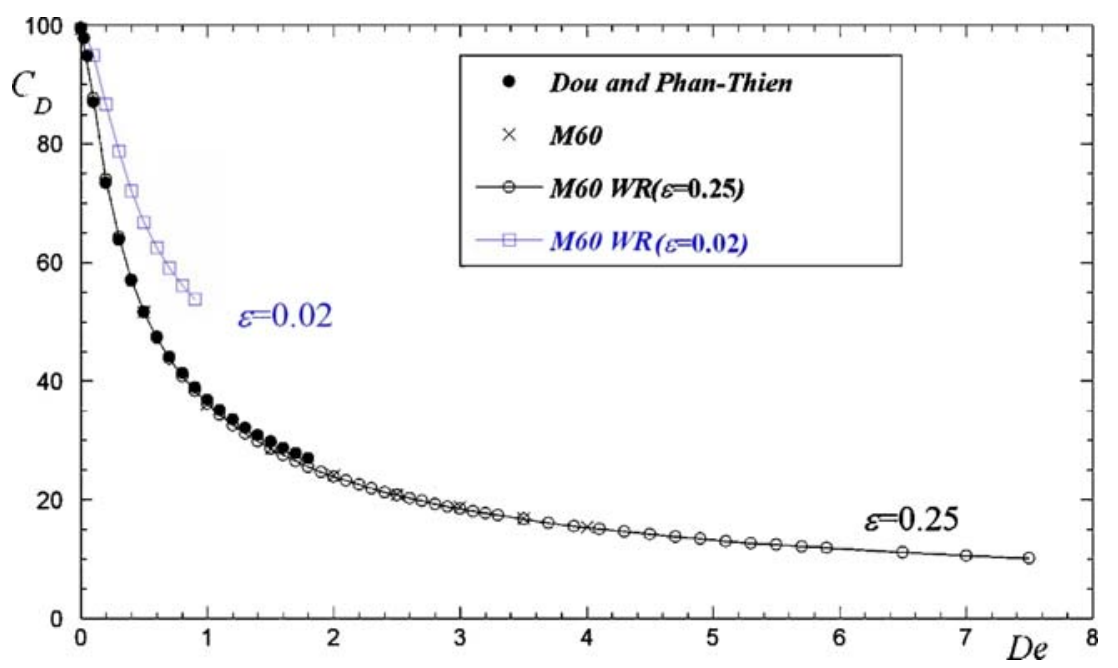

Kim et al. (2005a) for the two extensibility parameters considered, but values of $D e$ three times higher could be attained here.

Figure $12 \mathrm{a}$ and $\mathrm{b}$ show axial velocity and stress contour plots for the FENE-CR model with $\beta=0.1, D e=3.0$ at two values of extensibility, $L^{2}=10$ and $L^{2}=100$, respectively. For $L^{2}=100$ (Fig. 12a), i.e., when the $F$ term in Eq. 3 tends to unity and the FENE-CR model approaches the Oldroyd$\mathrm{B}$ model, the maximum values of the normal stress are located along the cylinder sidewall, due to shear flow and in the birefringent strand in the centreline downstream of the cylinder, here due to extensional flow. The birefringent strand at higher value of extensibility $L^{2}=100$ is much longer and exhibits higher stress values than the corresponding case with a lower value of extensibility, $L^{2}=10$, as shown in Fig. 12b. In both cases, the fore-aft asymmetry is more pronounced than was the case with the previous models (cf. Fig. 3), and a negative wake downstream of the cylinder is now visible (contours of $u / U>1)$.

As briefly discussed in the "Introduction" section, the onset and strength of the negative wake depends strongly on the extensibility parameter $L^{2}$ of the FENE-CR model, and for uniform approach flow, several authors (Satrape and Crochet 1994, Harlen 2002 and Dou and Phan-Thien 2004) indicated the absence of a negative wake for high values of $L^{2}$, as the fluid behaviour approaches that of the Oldroyd-B model. Dou and Phan-Thien (2003) suggested that the absence of negative wake in the experiments of McKinley et al. (1993) can be attributed to the high extensibility of the fluid (which was modelled with the FENE-CR having $L^{2}=144$ based on rheological measurements). This trend is also observed in our results, when comparing Fig. 13a and b. For the FENE-CR model with $L^{2}=100$ (Fig. 13a), and for small values of $D e(<1.5)$, no negative wake is observed. Only upstream, followed by downstream shifts $(0.5<D e<1.5)$ in the velocity profiles are observed with respect to the Newtonian case, and these variations are similar to those reported for the Oldroyd-B fluid in Fig. 10. Then, for $D e>1.5$ there is also an initial small upstream shift, followed by a downstream shift extending to $x / R \approx 4.5$, and finally, a negative wake appears that extends further downstream to $x / R \approx 16$. The magnitude of the negative wake, measured by the relative

Table 5 Drag force coefficient for the PTT model

\begin{tabular}{|c|c|c|c|}
\hline \multirow[t]{2}{*}{$D e$} & \multicolumn{2}{|c|}{ PTT $\varepsilon=0.25$} & \multirow{2}{*}{$\frac{\text { PTT } \varepsilon=0.02}{\mathrm{M} 60_{\mathrm{WR}}}$} \\
\hline & M60 & $\mathrm{M} 60_{\mathrm{WR}}$ & \\
\hline 0.1 & & 87.672 & 94.948 \\
\hline 0.2 & & 74.035 & 86.716 \\
\hline 0.3 & & 64.257 & 78.760 \\
\hline 0.4 & & 57.133 & 72.102 \\
\hline 0.5 & 51.681 & 51.690 & 66.814 \\
\hline 0.6 & & 47.370 & 62.540 \\
\hline 0.7 & & 43.839 & 59.115 \\
\hline 0.8 & & 40.886 & 56.147 \\
\hline 0.9 & & 38.372 & 53.763 \\
\hline 1.0 & 36.227 & 36.202 & \\
\hline 1.5 & 28.655 & 28.584 & \\
\hline 2.0 & 24.081 & 23.936 & \\
\hline 2.5 & 20.970 & 20.774 & \\
\hline 3.0 & 18.788 & 18.462 & \\
\hline 3.5 & 16.836 & 16.713 & \\
\hline 4.1 & & 15.082 & \\
\hline 5.1 & & 13.061 & \\
\hline 6.5 & & 11.151 & \\
\hline 7.0 & & 10.626 & \\
\hline 7.5 & & 10.158 & \\
\hline
\end{tabular}


velocity overshoot $\Delta u / U=\left(u-u_{\text {Newt }}\right) / U$, increases with $D e$ and is approximately $11.7 \%$ at $D e=5.0$. When the extensibility parameter is reduced, the wake behaviour changes significantly, as illustrated in Fig. 13b. Now, for all values of $D e$, no initial upstream shift is observed, and the negative wake appears earlier at $D e>0.5$, after a small downstream shift of the velocity profile near the rear stagnation point. The magnitude of the negative wake is higher than for the $L^{2}=100$ case, increasing with $D e$ from approximately $1 \%$ at $D e=1.0$ to $32.8 \%$ at $D e=7.8$. However, the negative wake is shorter for $L^{2}=10(x / R \approx$ 10) than for $L^{2}=100(x / R \approx 16)$.
Fig. 15 a $\tau_{x x}$ and $\mathbf{b} u$ centreline profiles for the PTT model $(\varepsilon=0.25)$. The inset shows the wake velocity difference, $\Delta u / U=\left(u-u_{\text {Newt }}\right) / U$ a

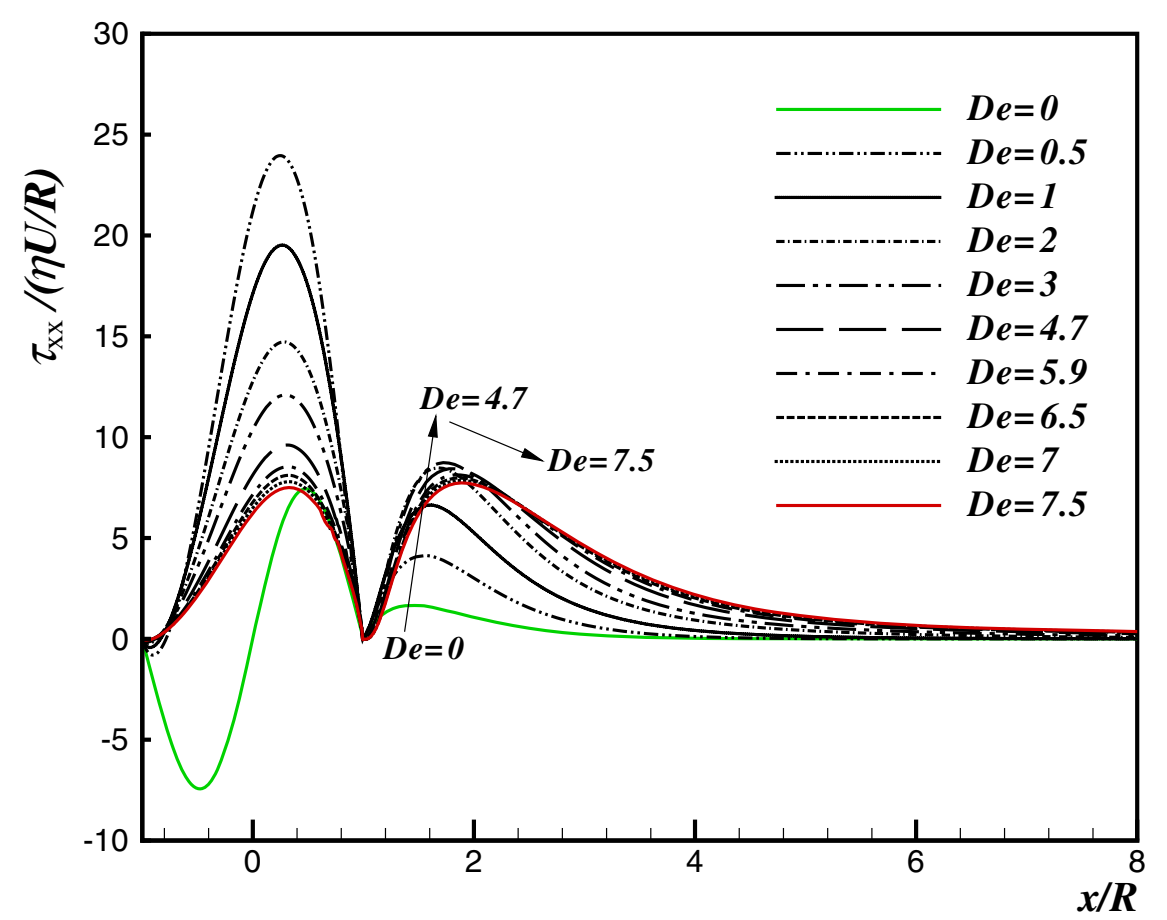

b

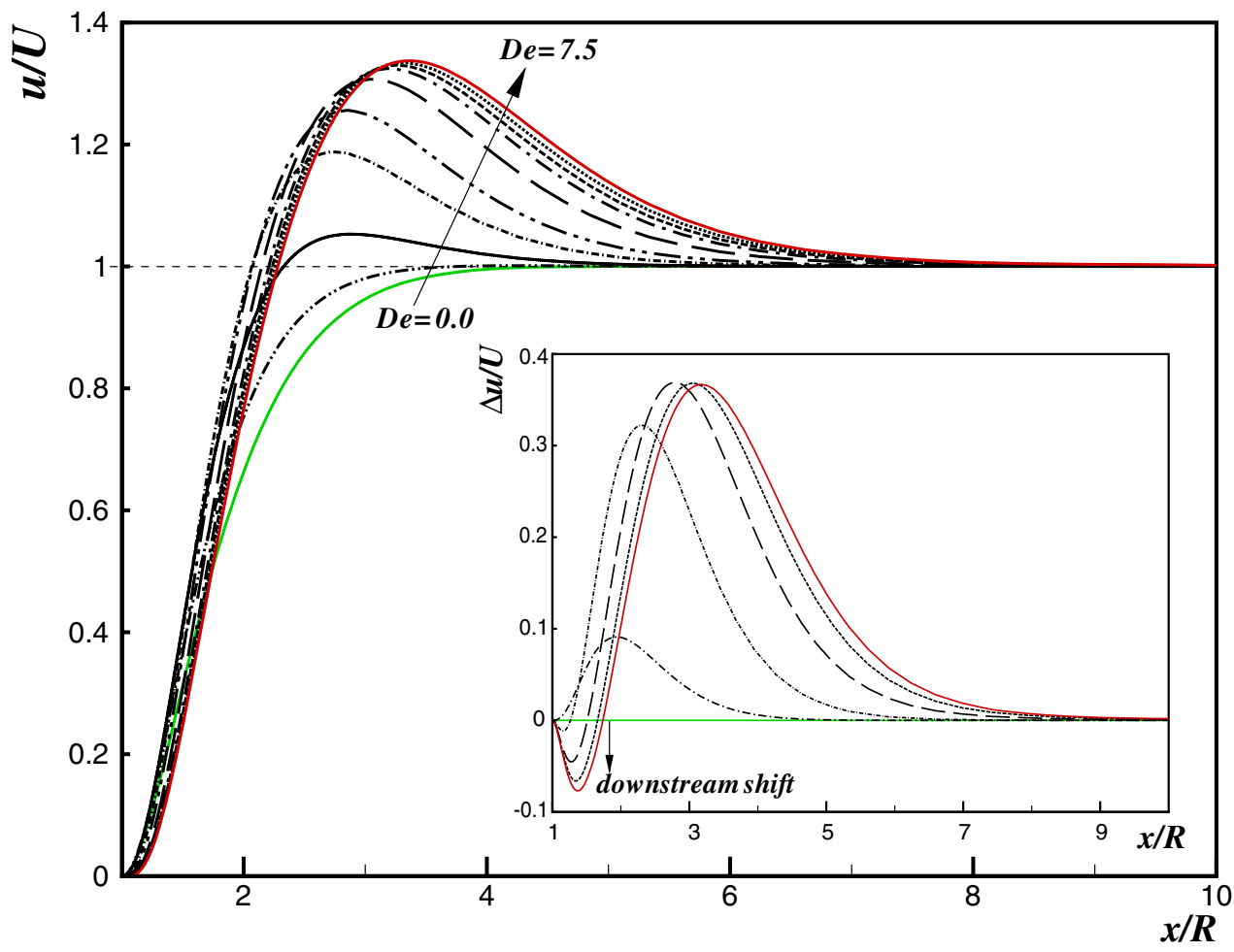




\section{PTT Model}

The PTT model includes the stress coefficient function, see Eq. 5, bringing in a new parameter $\varepsilon$ that imposes an upper limit to the elongational viscosity $\left(\eta_{\mathrm{E}}-1 / \varepsilon\right.$ for low $\left.\varepsilon\right)$.
This model is shear-thinning in viscosity, in contrast with the FENE-CR of the previous section, and was used in several numerical works of flow past a cylinder with both uniform and Poiseuille inlet conditions (Phan-Thien and Dou 1999 and Dou and Phan-Thien 2003). Here, the PTT
Fig. 16 a $\tau_{x x}$ and $\mathbf{b} u$ centreline profiles for the PTT model $(\varepsilon=0.02)$. The inset shows the wake velocity difference, $\Delta u / U=\left(u-u_{\text {Newt }}\right) / U$ a

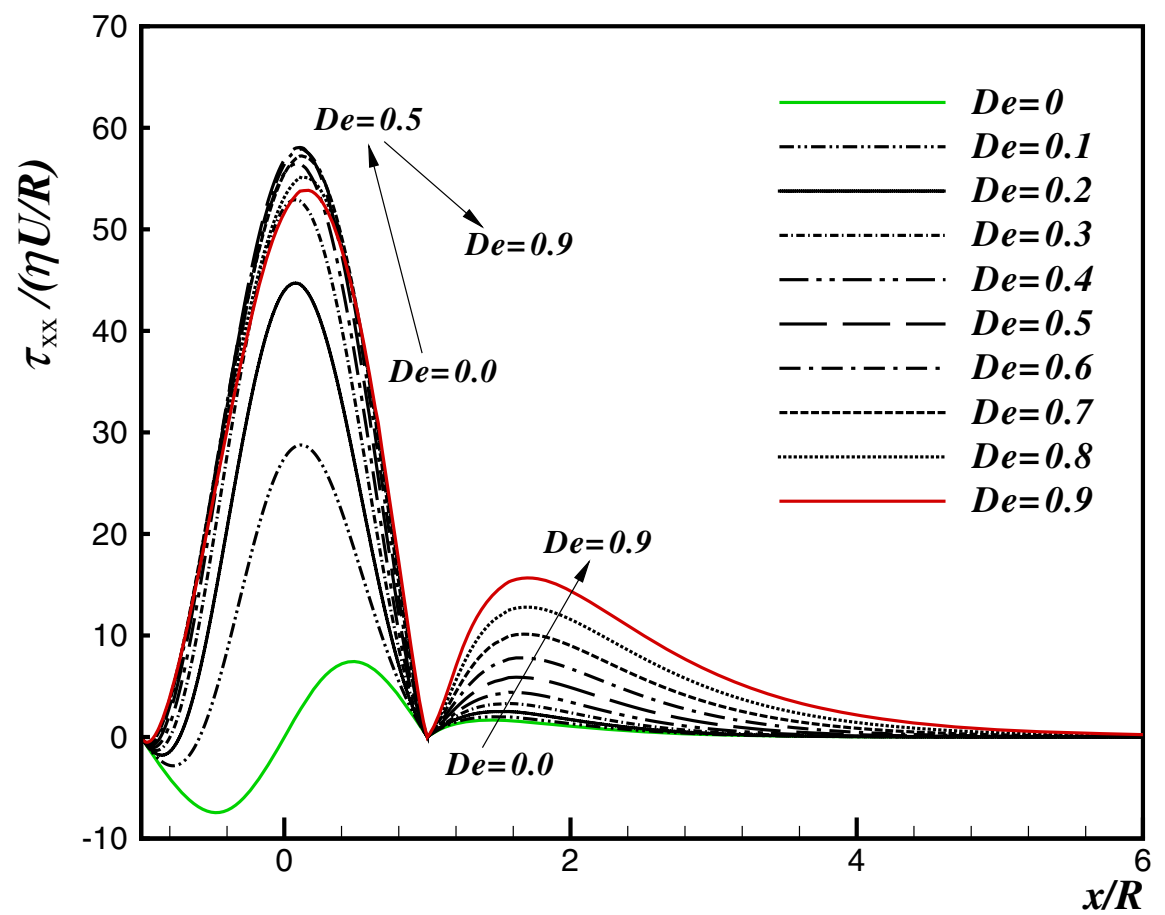

b

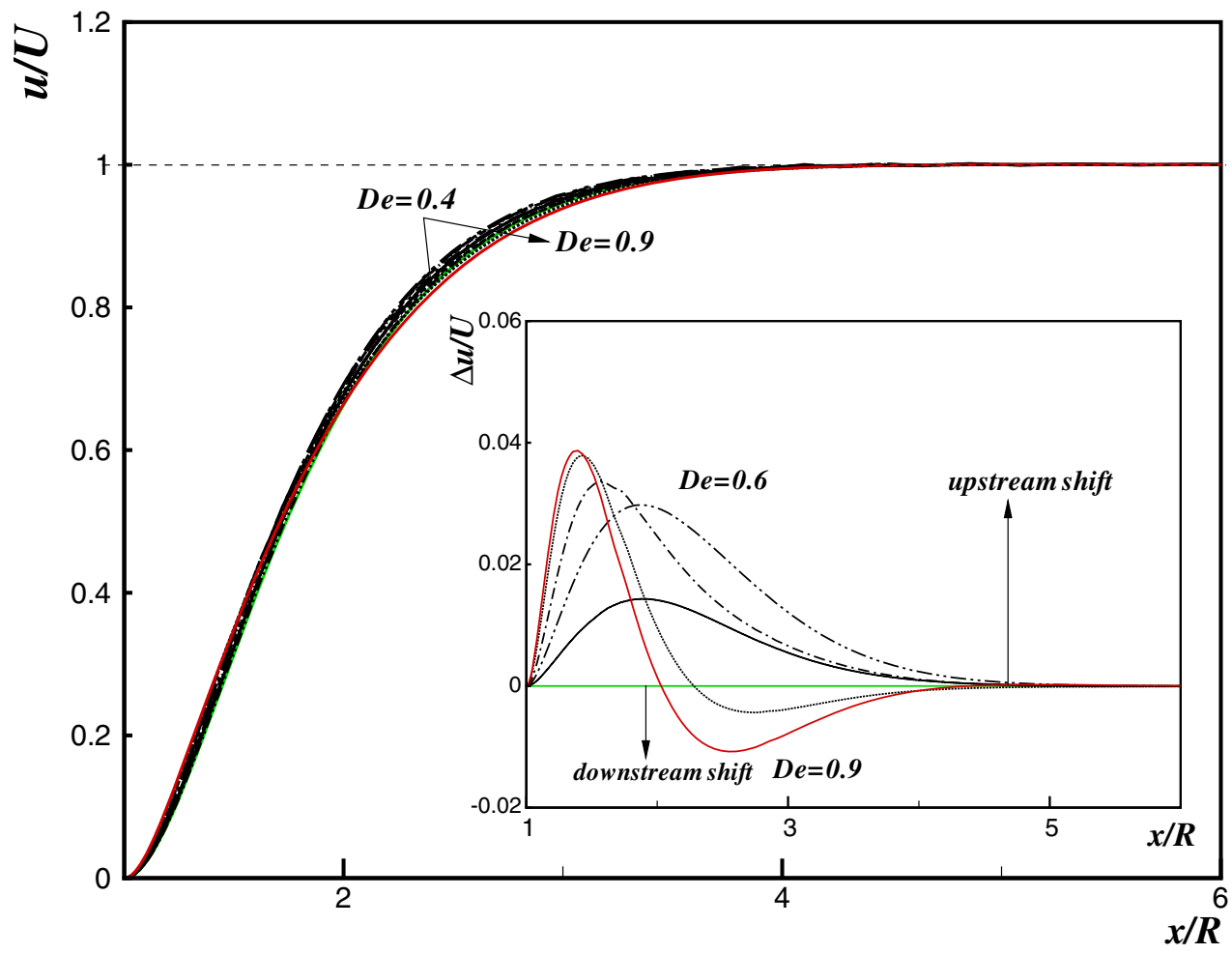


Fig. 17 Drag force coefficient for a Giesekus fluid $(\alpha=0.02$ and $\beta=0.59$ ). Mesh $\mathrm{M} 60_{\mathrm{WR}}$ $(-0-)$ and M60 (×)

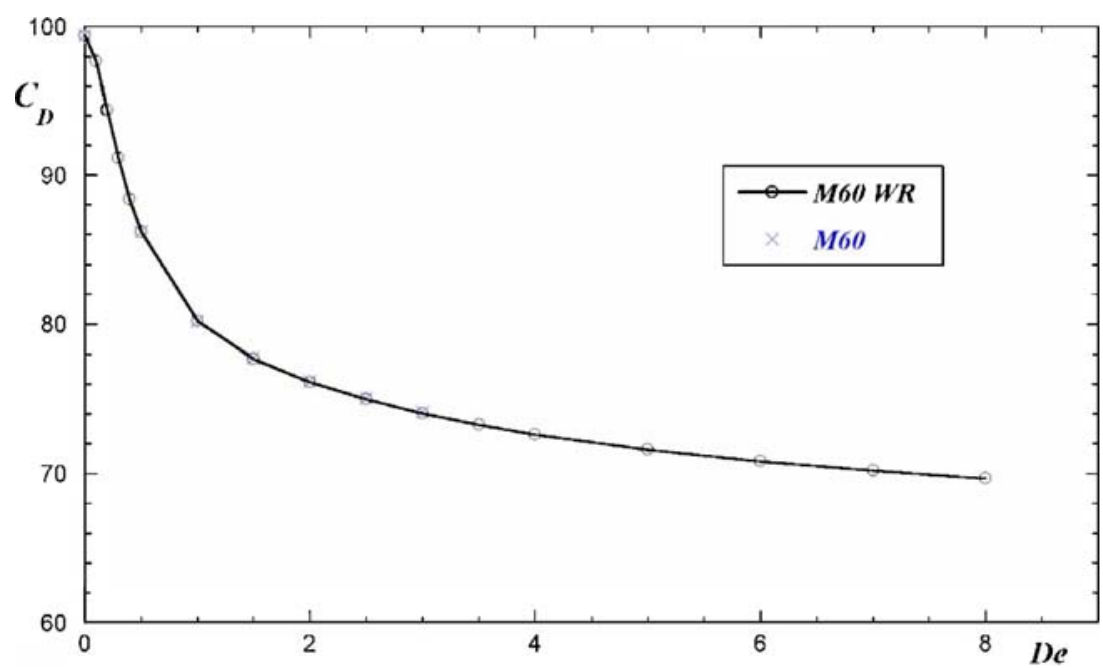

model is employed without a solvent viscosity $(\beta=0)$, for two typical parameters $\varepsilon=0.02$ and 0.25 . Note that when $\varepsilon \rightarrow 0$ the PTT model approaches the UCM model.

Figure 14 compares the computed $C_{\mathrm{D}}$ values with those obtained by Dou and Phan-Thien (2003). The present predictions obtained on meshes $\mathrm{M} 60$ and $\mathrm{M} 60_{\mathrm{WR}}$ are virtually indistinguishable and show the drag coefficient (listed in Table 5) to decrease monotonically with the Deborah number, up to limiting values of $D e=0.9$ and $D e=$ 7.5 , for $\varepsilon=0.02$ and $\varepsilon=0.25$, respectively. Generally, numerical simulations are easier, the higher the value of $\varepsilon$ and the lower the Deborah number, and this is borne out in the good agreement observed in Fig. 14 for $\varepsilon=0.25$ against the results of Dou and Phan-Thien (2003), to be contrasted with the poor comparison involving constant viscosity models $(\varepsilon=0.0)$ in Fig. 6. Above $D e \approx 0.9$, our results are below those of Dou and Phan-Thien (2003) and furthermore we could attain a maximum Deborah number four times higher.

Figures 15 and 16 present our predictions of axial normal stress and streamwise velocity along the cylinder wall and rear centreline for the two cases $\varepsilon=0.25$ and $\varepsilon=$ 0.02 , respectively. Generally speaking, the stress levels are much lower than those of previous models on account of shear-thinning which affects both the shear viscosity and the normal stresses. For the PTT model with the higher value of extensional parameter $(\varepsilon=0.25)$ the $\tau_{x x}$ predictions present some features not previously seen. Figure $15 \mathrm{a}$ shows that on the cylinder sidewalls for $D e>0.5$, all $\tau_{x x}$ profiles decrease with an increase of Deborah number due to shear-thinning, this decrease being more intense at smaller $D e$ values. For small values of $D e$, not shown here, the profiles of $\tau_{x x}$ exhibit a progressive increase with $D e$, these corresponding to situations where the shear-thinning is very weak and the effect of elasticity prevails. This is actually seen to a larger extent in Fig. 16a where the low value of $\varepsilon$ imparts a weaker shear-thinning. Above $D e \approx 5$ the decrease in the normal stress peak at the cylinder sidewalls is not so intense, with a constant maximum normalized value of approximately 7 to 8 . In the near wake region, the $\tau_{x x}$ profiles increase with $D e$ up to $D e \approx 5$, and then a small decrease and shift to downstream locations occurs for higher $D e$ values.

For the PTT model with a low value of $\varepsilon(\varepsilon=0.02)$, the behaviour of the $\tau_{x x}$ profiles is akin to that obtained with the UCM model (cf. Figs. 16a and 8a). The $\tau_{x x}$ profiles increase with $D e$ up to $D e \approx 0.5$ and then decrease up to the critical value $(D e=0.9)$. In the rear wake zone, there is a slow monotonic increase in $\tau_{x x}$ on account of extensional effects and the small amounts of shear-thinning.

Table 6 Drag force coefficient for the Giesekus model

\begin{tabular}{lll}
\hline Giesekus & & \\
\hline De & M60 & M60 \\
\hline 0.1 & & 97.672 \\
0.2 & & 94.394 \\
0.3 & & 91.151 \\
0.4 & & 88.401 \\
0.5 & 86.190 & 86.178 \\
1.0 & 80.217 & 80.198 \\
1.5 & 77.739 & 77.713 \\
2.0 & 76.192 & 76.160 \\
2.5 & 75.040 & 75.002 \\
3.0 & 74.114 & 74.063 \\
3.5 & & 73.300 \\
4.0 & & 72.652 \\
5.0 & & 71.623 \\
6.0 & & 70.837 \\
7.0 & & 70.201 \\
8.0 & & 69.684 \\
\hline
\end{tabular}


As expected, the formation of a negative wake depends on the $\varepsilon$ parameter, with an absence of this flow feature for low values of $\varepsilon$. These behaviours are illustrated by our results presented in Figs. 15b and 16b. The formation of a negative wake for $D e \geq 1$ with $\varepsilon=0.25$ is obvious in Fig. 15b, as well as a small downstream shift in the velocity profiles with respect to the Newtonian condition near the rear stagnation point. The negative wake seen with $\varepsilon=0.25$ extends to approximately $x / R \approx 9$ for $D e=7.5$. The relative velocity overshoot increases with $D e$ up to $D e \approx 5$, then asymptotes to a constant value of about $34 \%$ and shifts further downstream. With the reduction of the parameter $\varepsilon$, the flow behaviour becomes similar to that seen with the UCM model, as observed in Fig. 16b. For $\varepsilon=0.02$ there is an
Fig. 18 a $\tau_{x x}$ and $\mathbf{b} u$ centreline profiles for the Giesekus model $(\alpha=0.02$ and $\beta=0.59)$. The inset shows the wake velocity difference, $\Delta u / U=\left(u-u_{\text {New }}\right) / U$ a

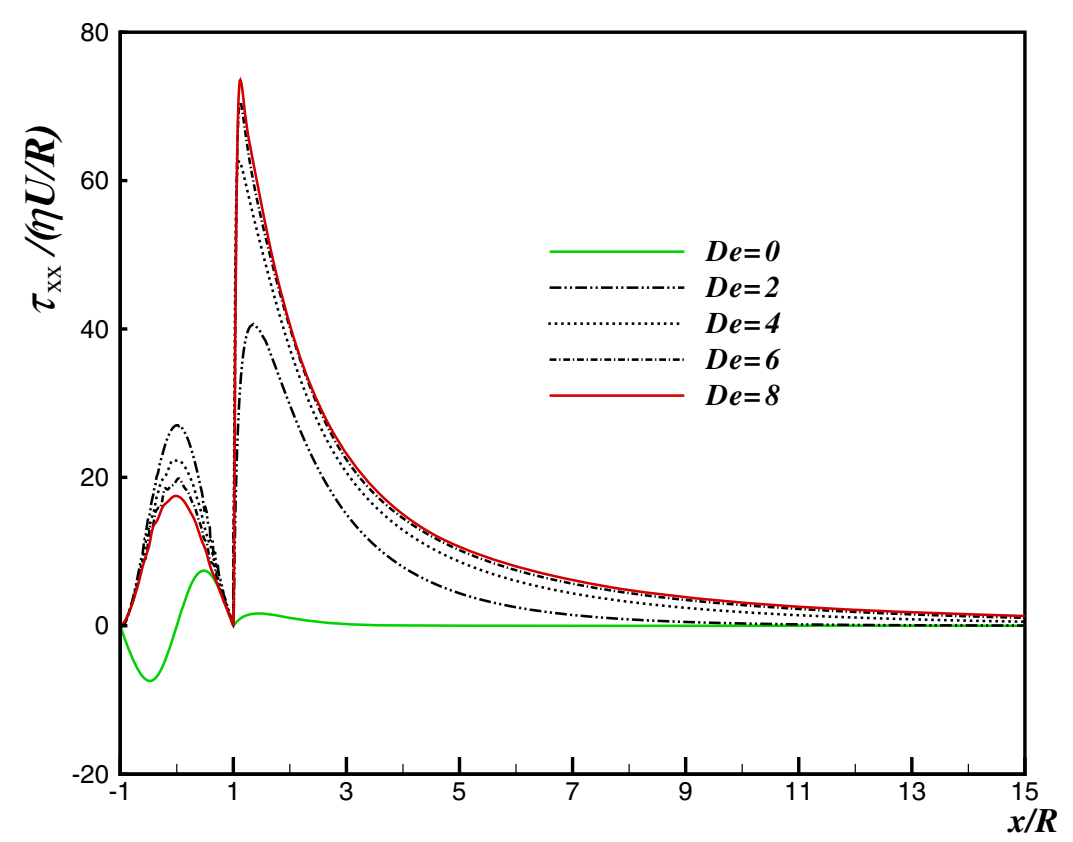

b

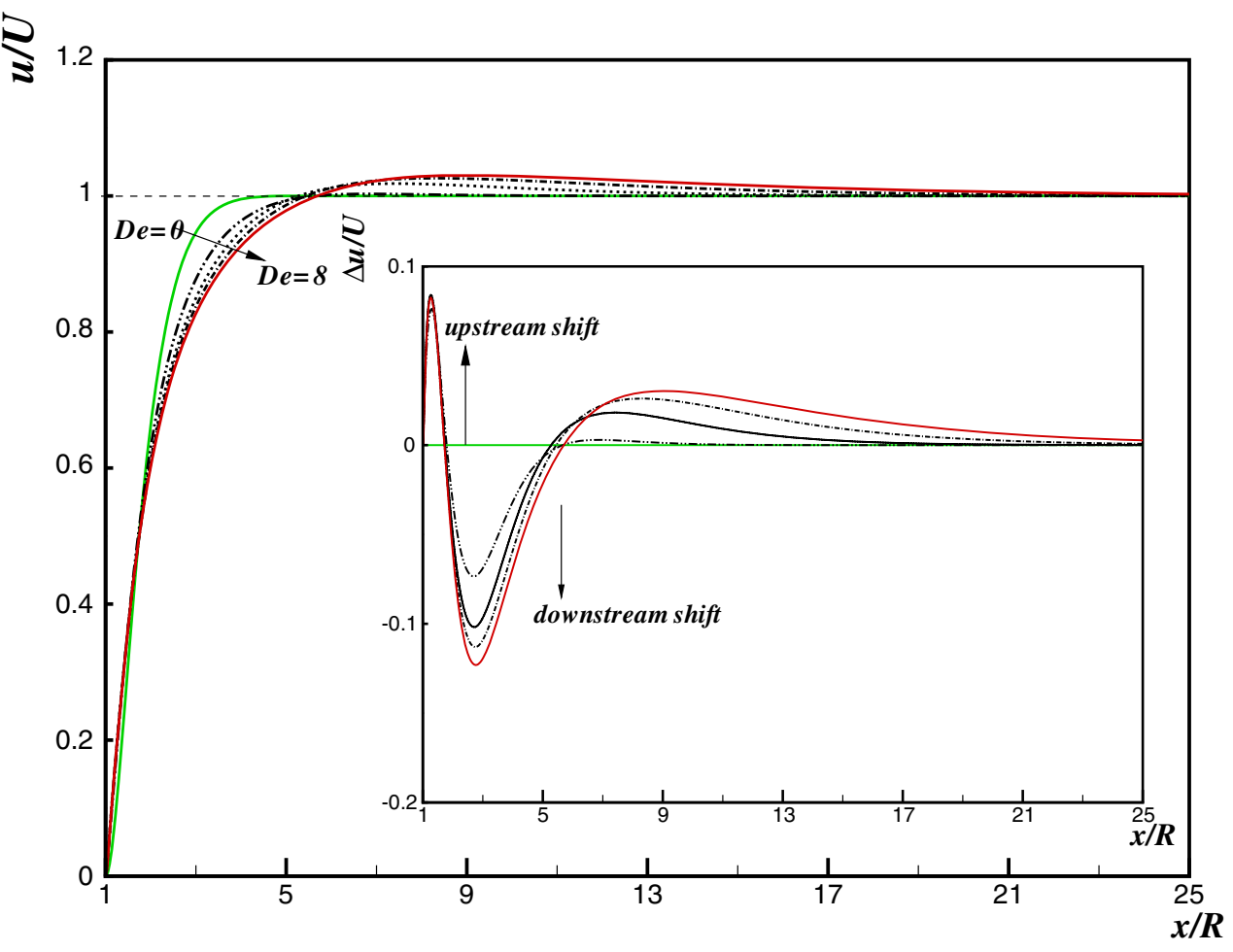


upstream shift in the velocity profiles with respect to the Newtonian profile up to $D e \approx 0.6$. At higher Deborah numbers, the velocity profiles also exhibit an upstream shift near the stagnation point in the rear wake of the cylinder, followed by a downstream shift further downstream of the cylinder, but no negative wake appears regardless of the value of $\varepsilon$.

\section{Giesekus model}

In this section, we discuss the results obtained with the Giesekus model for $\alpha=0.02$ and $\beta=0.59$. This viscosity ratio, $\beta$, is frequently used in numerical works and can be traced back to the value adopted in the experimental work of McKinley et al. (1993) for the Boger fluid used in their experiments. Hulsen et al. (2005) applied an implementation of the log-conformation methodology with a finite element method to the benchmark flow of Oldroyd-B and Giesekus fluids past a fixed confined cylinder. An almost unbounded convergence limit for the Giesekus model was reported, whereas for the Oldroyd-B, the solution became unsteady at high Deborah numbers while exhibiting symptoms of mesh dependency.

Figure 17 presents the $C_{\mathrm{D}}$ values obtained with meshes $\mathrm{M} 60$ and $\mathrm{M} 60_{\mathrm{WR}}$ for the Giesekus model (quantified in Table 6). The drag force coefficient decreases monotonically with the Deborah number, and convergent simulations could be obtained up to $D e \approx 8$. A direct comparison cannot be made against the results of Hulsen et al. (2005) because these are for the flow around a fixed cylinder, but it is clear that the log-conformation formulation offers a much larger range of allowable Deborah numbers.
Figure 18a shows the $\tau_{x x}$ profiles for the Giesekus model, where it can be observed that along the cylinder sidewalls, all profiles decrease with increasing Deborah number $(D e \geq 2)$, again a consequence of shear-thinning. As for the PTT case, this decrease is more intense at low Deborah numbers (up to $D e \approx 6$ ) than at higher De. On the other hand, in the rear wake zone, the $\tau_{x x}$ profiles increase for all $D e$, and the maximum value is greater than in the cylinder sidewalls above $D e=1$. Although the simulations were stable and convergent, some oscillations in the stress profiles are observed in the cylinder sidewalls and in the rear wake zone at high $D e$.

The formation of a negative wake at $D e \geq 1$ is also seen for the Giesekus model in Fig. 18b, where initially an upstream shift in the velocity profiles, with respect to the Newtonian profile, is present near the rear stagnation point and is followed by a downstream shift that extends to approximately $x / R \approx 5$. The length of the negative wake increases with $D e$ so that at $D e=8$, it extends in the axial direction up to $x / R \approx 25$. Even though the relative velocity overshoot increases with $D e$, its magnitude is not as large as seen with the previous models, attaining a maximum value of only about $3 \%$ at $D e=8$.

\section{Conclusions}

In this work, we present detailed results of a numerical investigation of the flow of viscoelastic fluids past a confined cylinder settling between two parallel plates with a $50 \%$ blockage ratio, using a finite-volume method. To properly assess the effect of various rheological properties,
Fig. 19 Maximum negative wake magnitude $\left(u_{\max }-U\right) / U$ (\%) for the FENE-CR, PTT and Giesekus models

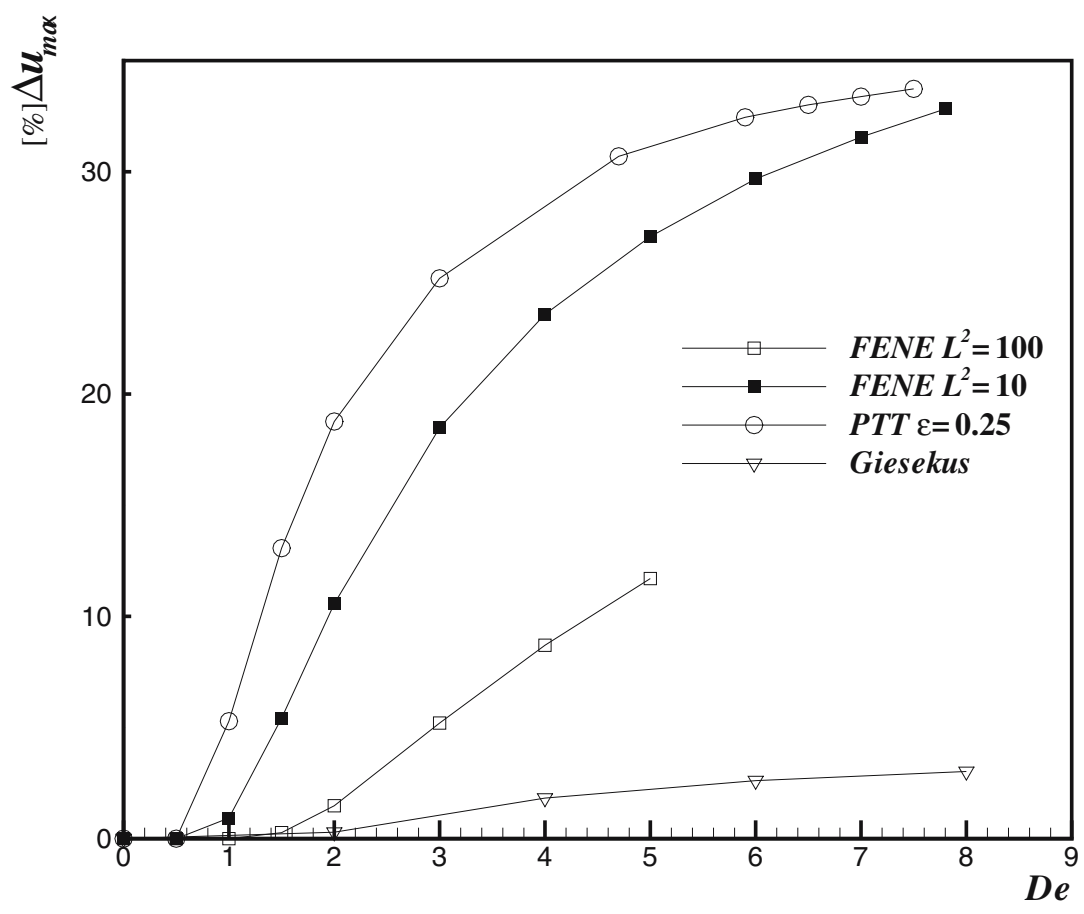


five constitutive models have been used: UCM, Oldroyd-B, FENE-CR, PTT and Giesekus models.

Simulations were carried out under creeping flow conditions, using very fine meshes, especially in the wake of the cylinder where large normal stress gradients are observed for high Deborah number flows. Numerical solutions could be obtained up to Deborah numbers in excess of those reported previously in the literature, especially in the case of the PTT, FENE-CR and Giesekus models. Special care was exercised to guarantee that all solutions are well converged iteratively, with stopping tolerances of around $10^{-4}$ and that sufficient mesh convergence is also provided, with smaller mesh spacing of order $\Delta r=0.002$, allowing estimation of drag coefficients which are exact up to the first decimal place, corresponding to an accuracy of around $0.1-0.3 \%$ on average. Besides providing reliable data for the drag coefficient variation and graphs of velocity and normal stress profiles along the wake, we reached some other conclusions worth mentioning:

1. Drag coefficient: For the UCM and the Oldroyd-B fluids with low solvent viscosity contribution $(\beta=$ 0.125 ), the drag coefficient on the cylinder decreases monotonically with increasing Deborah number. When the solvent viscosity contribution is high $(\beta=0.8)$, the drag coefficient first decreases with $D e$ followed by a levelling out and marginal, very slight increase, for $D e \geq 1.1$. For all retardation ratios, our results are well below the predictions of Dou and Phan-Thien (2003), with agreement only between the Newtonian cases up to $D e \approx 0.3$. For the other models with bounded extensional viscosity, the FENE-CR, PTT and Giesekus, the latter two being shear-thinning, the drag coefficient decreases monotonically with increasing Deborah number. For the FENE-CR model, good agreement was found with the $C_{\mathrm{D}}$ results of Kim et al. (2005a) for both extensibility parameters, $L^{2}=10$ and $L^{2}=100$, whereas the predictions of Dou and PhanThien (2003) with $L^{2}=10$ lie well below both sets of results, close to the predictions for the $L^{2}=100$ case. For $L^{2}=100$, the results from Dou and Phan-Thien (2003) show a non-monotonic behaviour, diverging from both our predictions and those of Kim et al. (2005a), with agreement only for $D e \leq 0.6$.

2. Negative wake: Regarding the existence of negative wake, these sets of simulations have shown that models with unbounded or bounded but very large extensional viscosities do not exhibit this feature. Figure 19 summarizes our findings on the maximum magnitude of the negative wake for all models tested. For the UCM and Oldroyd-B fluids, there was no sign of a negative wake regardless of the values of $\beta$, with the velocity profiles presenting an upstream shift close to the back of the cylinder $(x / R \leq 1.5-2)$ followed by a downstream shift for $D e>0.5$. For the FENE-CR model with $L^{2}=100$, there is a negative wake for $D e>1.5$, starting about 3 radii from the rear of the cylinder and extending up to $x / R \approx 16$. The magnitude of the negative wake increases with $D e$ and is approximately $11.7 \%$ at $D e=5.0$, as seen in Figure 19. For the FENE-CR fluid with $L^{2}=10$, there is no upstream shift of the velocity, and the negative wake is more intense and clearly marked appearing at $D e>0.5$, and formed at about one radius distance behind the cylinder. For $L^{2}=10$, the magnitude of the negative wake is larger than for $L^{2}=$ 100 , being $32.8 \%$ at $D e=7.8$, whereas its length is actually smaller, with $x / R \approx 10$ for $L^{2}=10$ and $x / R \approx 16$ for $L^{2}=100$. For the PTT fluid with the highest elongational parameter $\varepsilon=0.25$, a negative wake in the velocity overshoot is found at $D e>0.5$, starting at $x / R \approx 2$ and extending to approximately $x / R \approx 9$. The relative velocity overshoot increases with $D e$ up to $D e \approx 5$ and then asymptotes to a constant value of about $34 \%$, while its location shifts further downstream, as seen in Fig. 15. For lower values of the $\varepsilon$ parameter, the flow behaviour becomes similar to that seen with the UCM model. The Giesekus model produces an initial upstream shift followed by a downstream shift and a negative wake for $D e \geq 2$, starting at $x / R \approx 5.5$ and extending in the axial direction up to $x / R \approx 25$. The negative wake magnitude increases with $D e$, attaining a maximum value of about $3 \%$ for $D e=8$.

Acknowledgements The authors acknowledge funding from FEDER and FCT through projects POCI/EQU/59256/2004, POCI/ EQU/56342/2004 and POCI/EME/59338/2004. A. Afonso would also like to thank FCT for financial support through the scholarship SFRH/ BD28828/ 2006. We appreciate helpful conversations with Dr. R. J. Poole and thank him for proofreading this manuscript.

\section{References}

Alves MA, Pinho FT, Oliveira PJ (2000) Effect of a high resolution differencing scheme on finite-volume predictions of viscoelastic flows. J Non-Newt Fluid Mech 93:287-314

Alves MA, Pinho FT, Oliveira PJ (2001) The flow of viscoelastic fluids past a cylinder: finite-volume high-resolution methods. J Non-Newtonian Fluid Mech 97:207-232

Alves MA, Oliveira PJ, Pinho FT (2003) A convergent and universally bounded interpolation scheme for the treatment of advection. Int J Num Meth Fluids 41:47-75

Arigo MT, McKinley GH (1998) An experimental investigation of negative wakes behind spheres settling in a shear thinning viscoelastic fluid. Rheol Acta 37:307-327

Baaijens FPT (1998) Mixed finite element methods for viscoelastic flow analysis: a review. J Non-Newtonian Fluid Mech 79:361385 
Bird RB, Armstrong RC, Hassager O (1987) Dynamics of polymeric liquids, vol 1. Fluid mechanics, 2nd edn. Wiley, New York

Broadbent JM, Mena B (1974) Slow flow of an elastico-viscous fluid past cylinders and spheres. Chem Eng J 8:11-19

Bush MB (1993) The stagnation flow behind a sphere. J NonNewtonian Fluid Mech 49:103-122

Bush MB (1994) On the stagnation flow behind a sphere in a shearthinning viscoelastic liquid. J Non-Newtonian Fluid Mech $55: 229-247$

Caola AE, Joo YL, Armstrong RC, Brown RA (2001) Highly parallel time integration of vicoelastic flows. J Non-Newtonian Fluid Mech 100:191-216

Chilcott MD, Rallison JM (1988) Creeping flow of dilute polymer solutions past cylinders and spheres. J Non-Newtonian Fluid Mech 29:381-432

Coates PJ, Armstrong RC, Brown RA (1992) Calculation of steadystate viscoelastic flow through axisymmetric contractions with the EEME formulation. J Non-Newtonian Fluid Mech 42:141188

Dou HS, Phan-Thien N (2003) Negative wake in the uniform flow past a cylinder. Rheol Acta 42:383-409

Dou HS, Phan-Thien N (2004) Criteria of negative wake generation behind a cylinder. Rheol Acta 43:203-209

Dupret F, Marchal JM (1986) Loss of evolution in the flow of viscoelastic fluids. J Non-Newtonian Fluid Mech 20:143-171

Fan Y, Tanner RI, Phan-Thien N (1999) Galerkin/least-square finiteelement methods for steady viscoelastic flows. J Non-Newtonian Fluid Mech 84:233-256

Gaskell PH, Lau AKC (1988) Curvature-compensated convective transport: SMART a new boundedness preserving transport algorithm. Int J Numer Meth Fluids 41:617-641

Gerritsma MI (2006) Direct minimization of the discontinuous leastsquares spectral element method for viscoelastic fluids. J Sci Comp 27:245-256

Giesekus H (1982) A simple constitutive equation for polymer fluids based on the concept of deformation-dependent tensional mobility. J Non-Newtonian Fluid Mech 11:69-109

Harlen OG (2002) The negative wake behind a sphere sedimenting through a viscoelastic fluid. J Non-Newtonian Fluid Mech $108: 411-430$

Hassager O (1979) Negative wake behind bubbles in non-Newtonian liquids. Nature 279:402-403

$\mathrm{Hu} \mathrm{HH}$, Joseph DD (1990) Numerical simulation of viscoelastic flow past a cylinder. J Non-Newtonian Fluid Mech 37:347-377

Huang PY, Feng J (1995) Wall effects on the flow of viscoelastic fluids around a circular cylinder. J Non-Newtonian Fluid Mech 60:179-198

Hulsen MA (1988) Some properties and analytical expressions for plane flow of Leonov and Giesekus models. J Non-Newtonian Fluid Mech 30:85-92

Hulsen MA, Fattal R, Kupferman R (2005) Flow of viscoelastic fluids past a cylinder at high Weissenberg number: Stabilized simulations using matrix logarithms. J Non-Newtonian Fluid Mech $127: 27-39$
Jin H, Phan-Thien N, Tanner RI (1991) A finite element analysis of the flow past a sphere in a cylindrical tube: PTT fluid model. Comput Mech 8:409-422

Kim JM, Kim C, Ahn KH, Lee SJ (2004) An efficient iterative solver and high precision solutions of the Oldroyd-B fluid flow past a confined cylinder. J Non-Newtonian Fluid Mech 123:161-173

Kim JM, Kim C, Chung C, Ahn KH, Lee SJ (2005a) Negative wake generation of FENE-CR fluids in uniform and Poiseuille flows past a cylinder. Rheol Acta 44:600-613

Kim JM, Kim C, Kim JH, Chung C, Ahn KH, Lee SJ (2005b) Highresolution finite element simulation of 4:1 planar contraction flow of viscoelastic fluid. J Non-Newtonian Fluid Mech 129:2337

Liu AW, Bornside DE, Armstrong RC, Brown RA (1998) Viscoelastic flow of polymer solutions around a periodic, linear array of cylinders: comparisons of predictions for microstructure and flow fields. J Non-Newtonian Fluid Mech 77:153-190

Manero O, Mena B (1981) On the slow flow of viscoelastic liquids past a circular cylinder. J Non-Newtonian Fluid Mech 9:379-387

McKinley GH, Armstrong RC, Brown RA (1993) The wake instability in viscoelastic flow past confined circular cylinders. Philos Trans R Soc Lond A 344:265-304

Oliveira PJ, Pinho FT, Pinto GA (1998) Numerical simulation of nonlinear elastic flows with a general collocated finite-volume method. J Non-Newt Fluid Mech, 79:1-43

Oliveira PJ (2001) On the numerical implementation of nonlinear viscoelastic models in a finite-volume method. Num Heat Transfer Part B 40:283-301

Oliveira PJ, Miranda AIP (2005) A numerical study of steady and unsteady viscoelastic flow past bounded cylinders. J Non-Newtonian Fluid Mech 127:51-66

Owens RG, Chauviére C, Philips TN (2002) A locally-upwinded spectral technique (LUST) for viscoelastic flows. J Non- Newtonian Fluid Mech 108:49-71

Phan-Thien N, Tanner RI (1977) A new constitutive equation derived from network theory. J Non-Newtonian Fluid Mech 2:353-365

Phan-Thien N (1978) A non-linear network viscoelastic model. J Rheol 22:259-283

Phan-Thien N, Dou HS (1999) Viscoelastic flow past a cylinder: drag coefficient. Comput Meth Appl Mech Eng 180:243-266

Satrape JV, Crochet MJ (1994) Numerical simulation of the motion of a sphere in a Boger fluid. J Non-Newtonian Fluid Mech 55:91111

Sigli D, Coutanceau M (1977) Effect of finite boundaries on the slow laminar isothermal flow of a viscoelastic fluid around a spherical obstacle. J Non-Newtonian Fluid Mech 2:1-21

Sun J, Smith MD, Armstrong RC, Brown RA (1999) Finite element method for viscoelastic flows based on the discrete adaptive viscoelastic stress splitting and the discontinuous Galerkin method: DAVSS-G/DG. J Non-Newtonian Fluid Mech 86:281307

Zheng R, Phan-Thien N, Tanner RI (1991) The flow past a sphere in a cylindrical tube: effects of inertia, shear-thinning and elasticity. Rheol Acta 30:499-510 